\title{
Perspective
}

PERSPECTIVE Actualité en histoire de l'art

\section{1 | 2021}

Portugal et espaces lusophones

\section{La forme inattendue de soi}

Entretien avec Álvaro Siza, par Dominique Machabert

\section{Álvaro Siza et Dominique Machabert}

\section{OpenEdition}

Journals

Édition électronique

URL : https://journals.openedition.org/perspective/22968

DOI : $10.4000 /$ perspective.22968

ISSN : 2269-7721

Éditeur

Institut national d'histoire de l'art

\section{Édition imprimée}

Date de publication : 14 octobre 2021

Pagination : 104-129

ISBN : 978-2-917902-91-2

ISSN : $1777-7852$

Référence électronique

Álvaro Siza et Dominique Machabert, "La forme inattendue de soi », Perspective [En ligne], 1 | 2021, mis en ligne le 31 décembre 2021, consulté le 13 mai 2022. URL : http://journals.openedition.org/ perspective/22968; DOI : https://doi.org/10.4000/perspective.22968 



\title{
La forme inattendue de soi
}

\author{
Entretien avec Álvaro Siza, \\ par Dominique Machabert
}

\section{Álvaro Siza, l'autre}

Et comme il était vain d'y aller tout droit pour le cerner un peu ainsi que son travail, Fernando Távora ${ }^{1}$, son maître et ami, légende de l'école de Porto, était parti de l'autre. Attentif et, depuis fort longtemps, le premier à avoir vu " sous le front plein d'éminences $^{2}$ " la recherche encore ignorée du jeune homme, il avait dit de lui, bien après, qu'il était hollandais plus que les Hollandais lorsqu'il travaillait aux Pays-Bas et le plus allemand des architectes allemands à Berlin. Et, dans la circonstance, voyant son projet pour la Grande Bibliothèque de France, il avait pensé à Auguste Perret. Il voulait dire la capture dans l'air des traditions nationales, des cultures locales, des matériaux parfois injustement et inexplicablement délaissés. Il voulait dire la présence poreuse de la rumeur architectonique, la tension, la rue, le sentiment général pris à la volée, un monde d'indiscrétions et de trouvailles attrapé.

Je comprenais alors que c'était cela, être portugais : être hollandais, berlinois, capter jusqu'à ce quelque chose dans l'air de Paris, comme Auguste Perret ; être l'autre plus que l'autre ne saurait être lui-même, mieux qu'à sa propre place toujours un peu flottante, celle d'un Portugal qui se trouve avoir été, être durablement, plutôt dehors que dedans.

Au-delà d'une ouvre, Fernando Távora avait beaucoup occupé son temps à fonder un enseignement et à dire, ce faisant, qu'il y avait " sûrement une façon portugaise de faire ". Cela donnerait un courant d'abord un peu lointain, disons-le. Quelques indices simples, humbles et non définitifs, essentiels peuvent aider à s'en faire une idée, comme l'attention au site, l'adaptation aux circonstances immédiates et locales, une attention à l'intelligence des systèmes constructifs traditionnels, une approche du projet architectural plus pragmatique et artisanale que savante et technologique, une attention aux usages plus qu'aux fonctions sèches, une prégnance de la matérialité, le souci du détail, une économie de moyens, l'écoute de la rumeur sociale et du monde comme des grands courants d'architecture que l'on interprète, et le dessin, transport de la recherche en cours... Un courant parti à la fin des années 1940 à l'initiative de Carlos Ramos, alors directeur de ce qui était encore l'école des Beaux-Arts et deviendrait ensuite 
l'école de Porto. Pas de textes vraiment pour en dire les fondements, les orientations, la pédagogie, mais une transmission longtemps restée orale, inspirée et dessinée plutôt.

Alors que Pereira "prétend " chez Antonio Tabucchi" ${ }^{3}$ Siza ne prétend rien, ni même " la façon portugaise de faire ". Il y a chez lui une absence totale de prétention, laissant dire à son sujet et de son travail ce que l'on dit.

Pour Álvaro Siza, le mystère du projet architectural - entendons qu'il est aussi le sien - est dans les rapports : rapport de l'objet à lui-même, au site, à la ville immédiate et lointaine, au paysage, à l'histoire. Ses projets sont pris dans une constellation de rapports même insoupçonnés, dérisoires et fuyant, que dessiner sans relâche révèle et travaille jusqu'à toucher une qualité pareille à une présence, un instant, une trace, un signe, un mouvement dans l'air, une atmosphère comme il dit souvent, l'oubli même, qui n'en est pas moins une part active de plus ; pour que la situation projetée résulte non pas de l'effort qui l'a voulue mais de l'évidence fortuite de la trouver. Voilà qui serait Álvaro Siza, " architecte portugais ".

Voilà ce que je soutenais, qu'il ne partageait pourtant qu'à moitié, me laissant faire mais préférant à ce propos - et disant - n'être pas portugais, pas plus qu'autre chose, pas réductible au moins à cela. Ce qui pour moi revenait à dire le déplacement, toujours, ou l'autre. Ce qui, pour moi, revenait à dire Portugais.

[Dominique Machabert]

- Dominique Machabert. Comment interprétez-vous les propos de Fernando Távora lorsqu'il dit à votre propos "plus hollandais que les Hollandais aux Pays-Bas, plus allemand que les architectes allemands à Berlin »?

- Álvaro Siza. Je pense qu'il témoigne de son attachement profond pour ce qui fonde et enveloppe un lieu, une ville. Ce qu'on appelle aussi, pour faire vite, le contexte. Il est intéressant de noter chez Távora son intérêt pour l'actualité internationale la plus pointue en même temps qu'il fut un animateur très investi dans l'« Enquête ${ }^{4}$ » (fig. 1) après qu'il eut écrit un petit texte très important : "O problema da casa portuguesa ${ }^{5}$ » (" Le problème de la maison portugaise »). II y avait chez lui ce souci des racines ainsi qu'une vision large, internationale, de l'architecture. C'est probablement ce pour quoi il a été impressionné par mes travaux à

1. Arquitectura popular em Portugal (premières de couverture des deux volumes originaux), Lisbonne, Sindicato nacional dos arquitectos, 1961.
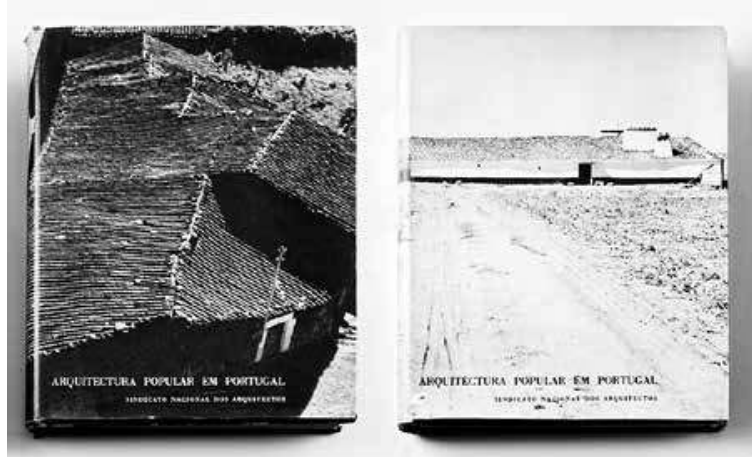
La Haye ou à Kreuzberg, à Berlin, allant même jusqu'à dire que mes bâtiments semblaient avoir toujours été là. C'était une appréciation généreuse et amicale de sa part. Mais elle faisait écho à ce qui importait particulièrement pour lui et qu'il n'a cessé de témoigner et d'enseigner.

- Dominique Machabert. Qu'est-ce que pouvait bien vouloir dire " être berlinois à Berlin » au point d'y réaliser un bâtiment qui « semble avoir toujours été là »?

- Álvaro Siza. Travailler dans une ville que je ne connaissais pas et que je découvrais pas à 


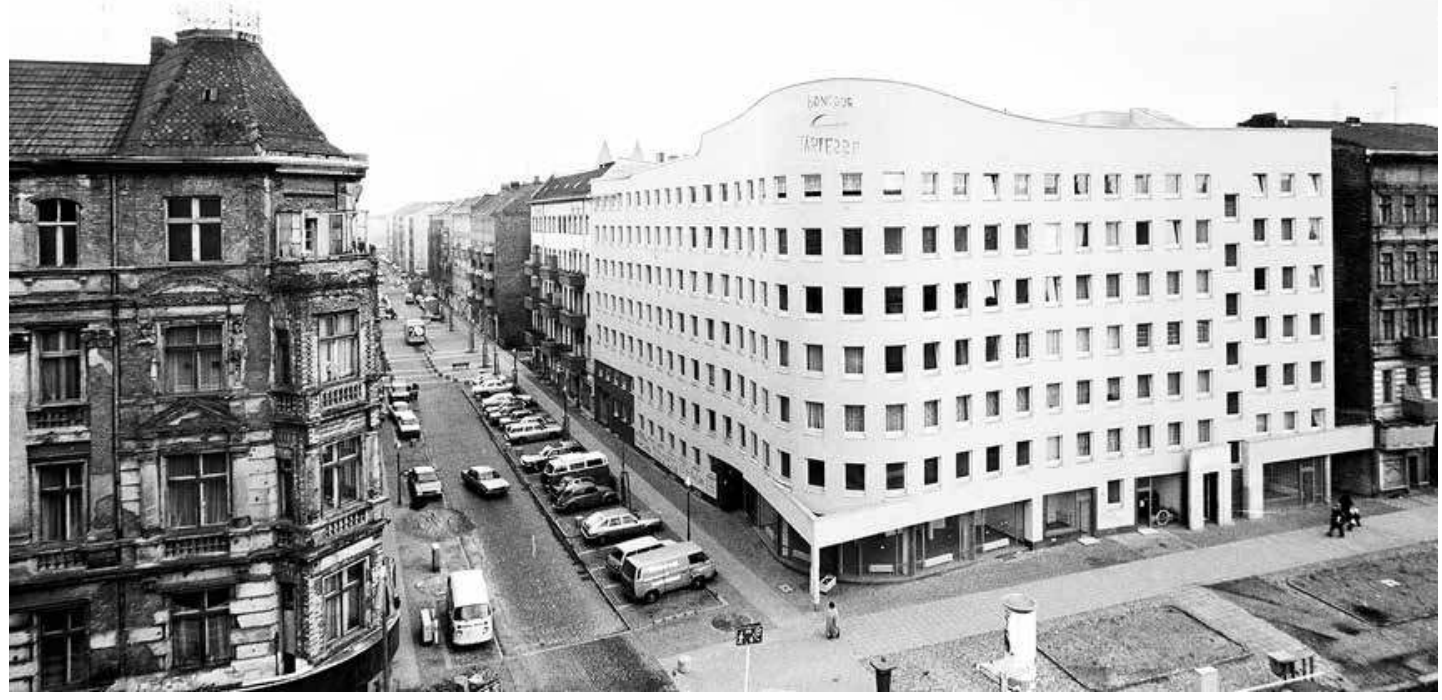

2a. Álvaro Siza, immeuble d'habitation Schlesisches Tor, dit «Bonjour Tristesse », Berlin 1982-1983, photographie de Giovanni Chiaramonte. pas m'avait apporté une énergie et un enthousiasme inouïs. Dans ces moments-là, les yeux captent tout, la tête attrape l'atmosphère. Depuis ce temps-là et comme récemment à New York pour un projet de tour (611 West 56th Street, 2015-), je ne commence jamais par analyser les villes en détail. Ma stratégie, quoique involontaire, consiste à aller au hasard par les rues sans appréhension, sans intention particulière ni programme très précis, au risque de me perdre, et à capter ainsi les choses, spontanément, sans calcul.

\section{- Dominique Machabert. Que capte-t-on quand on se perd à Berlin?}

- Álvaro Siza. Je me suis trouvé immergé dans ce que je ne connaissais alors que dans les livres. La naissance du mouvement moderne était là, sous mes yeux. À Berlin comme dans d'autres villes, mais à Berlin surtout, il y a cette tension née de la proximité entre expressionnisme et fonctionnalisme ; deux courants très forts, actifs et opposés, très idéologisés aussi, dans le climat particulièrement tendu des années 1930. Des quartiers « expressionnistes » et « rationalistes», proches les uns des autres et que tout oppose pourtant, forment ensemble un tissu continu. Me trouver dans une ville au coude à coude avec Ludwig Mies van der Rohe et Erich Mendelsohn, au cœur même de ce qui fut la naissance du mouvement moderne, de ses tensions, de ses contradictions, m'a beaucoup, beaucoup impressionné.

À Kreuzberg, je me souviens aussi de la sévérité et de l'austérité grise de ces quartiers datant du XVIII et du XIXe siècles, de la répétition des fenêtres, toujours les mêmes, et puis de l'angle des rues qui soudain fait l'effet d'une explosion de joie, d'une libération. Comme si, en ce point précis où l'on tourne, où l'on change de direction, l'angle venait soulager le cheminement éprouvé le long des hauts bâtiments et la répétition systématique des fenêtres. 


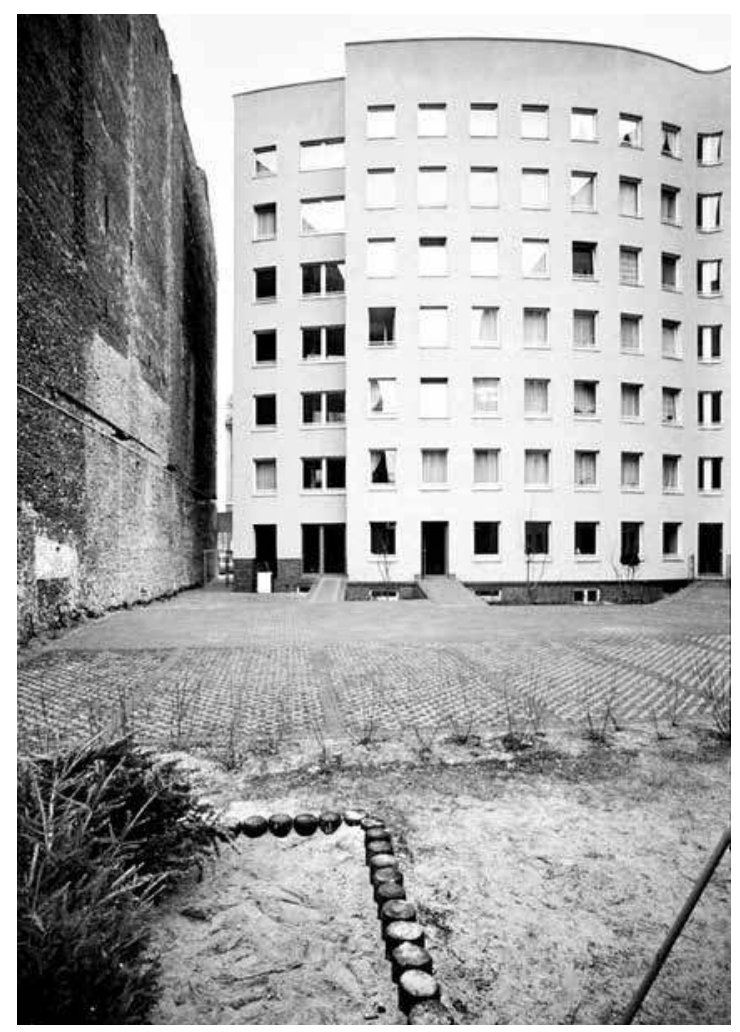

2b. Álvaro Siza, immeuble d'habitation Schlesisches Tor, Berlin, 1982-1983, photographie de Giovanni Chiaramonte.
L'angle est un hommage au mouvement, une bouffée d'air. On entre dans une rue " corridor » sans autre possibilité que de longer docilement les façades austères. Puis tout à coup, apparaît la possibilité d'une sortie! C'est très important ce qui se passe à partir de l'angle, élément absolument fondamental et basique de l'architecture, tant à l'intérieur d'un bâtiment qu'à l'extérieur et d'où partent le mouvement et les parcours ${ }^{6}$.

- Dominique Machabert. Il y a un angle à Kreuzberg rendu célèbre par votre bâtiment, l'immeuble d'habitation Schlesisches Tor (1982-1990 ; fig. 2a). Une main anonyme y a tagué l'inscription « Bonjour tristesse » qui finira par identifier l'édifice. Quelle signification donner à ce geste ?

- Álvaro Siza. C'était une période particulièrement délicate. Les immigrants turcs venus en nombre pour la reconstruction de Berlin après la guerre avaient peu à peu et naturellement pris leurs marques, à Kreuzberg surtout. L'ambiance, les magasins étaient turcs. Depuis la rue, venue des restaurants, on entendait de la musique turque. Et devant cet état de fait, des groupuscules d'Allemands particulièrement violents venaient manifester leur opposition, ce qui donnait lieu à de véritables batailles rangées.

- Dominique Machabert. Cette inscription disait donc une hostilité à l'égard du projet et de la philosophie de l'Internationale Bauausstellung (ou IBA) avec son vœu d'intégration et de participation des immigrés ${ }^{7}$ ?

- Álvaro Siza. L'intention était clairement hostile. Et puis la revue italienne Lotus a fait sa " une » avec le "Bonjour tristesse ». Et à partir de là, s'en est suivi un véritable feuilleton. En maintenant l'inscription notre intention n'était pas de faire un coup ou une performance. Mais nous n'avions pas d'autre choix que de la laisser car repeindre entièrement le bâtiment exigeait des moyens que nous n'avions pas.

Mais il y a plus important à noter concernant ce travail ainsi qu'un autre que je n'ai pas construit, à Kreuzberg aussi. On remarque qu'entre les bâtiments, dont une maison de retraite et une crèche que j'ai réalisées, nous avons ménagé des interstices. Les bâtiments ne se touchent pas. L'idée était de maintenir et de restaurer tout ce qui pouvait l'être. Et je n'avais aucunement l'intention de dénaturer le quartier avec les nouveaux bâtiments. Aussi j'ai veillé à favoriser un dialogue intense et rigoureux entre ce que nous maintenions et ce que nous construisions, ménageant entre les deux un espace très fin (fig. $\mathbf{2 b}$ ).

- Dominique Machabert. Concernant votre projet pour la Grande Bibliothèque de France à Paris (1989), Távora poursuit son observation faisant référence alors à Auguste Perret. Au sujet d'une tradition classique et moderne à la fois, c'est à la France qu'il pense. Qu'en dites-vous ? 
- Álvaro Siza. Távora vous a dit cela ? Je n'avais que la version courte, à propos de La Haye et de Berlin. Alors oui, je suis globalement d'accord avec ce qu'il dit à propos de Paris. J'ai beaucoup étudié les grands bâtiments qui bordent la Seine comme le Louvre, pas pour leur langage mais pour leur expression surtout. Notre bâtiment se présentait comme un front bâti de différents niveaux formant une sorte de barrière. Le projet prévoyait aussi un pont; ainsi, nous maintenions un lien très clair avec le fleuve. Finalement notre projet n'a pas été très bien reçu. Le seul à ma connaissance à en avoir dit du bien, c'est Ricardo Bofill. Au cours d'un de mes passages à Barcelone, il n'y a pas très longtemps d'ailleurs et bien des années après le concours, il m’a dit : «Ton projet pour la bibliothèque à Paris... chapeau! »Concernant Auguste Perret situé entre la tradition classique et le moderne, j'assume totalement la référence.

- Dominique Machabert. Qu'est-ce qui pousserait à dire de Perret qu'il serait une, voire «la » référence française ? Vous en parlez d'ailleurs dans votre discours pour la cérémonie de remise du Grand Prix d'architecture de l'Académie des beaux-arts, prononcé en octobre $2019^{8}$.

- Álvaro Siza. C'est probablement le plus important des modernes. Il a été une référence pour tous, pour Le Corbusier aussi. Qu'ils l'aient approché ou pas, tous lui doivent beaucoup.

Ce que l'œuvre de Perret nous dit, c'est la capacité d'assimilation - et avec quel brio ! - de I'architecture française. Serez-vous d'accord avec moi si je dis que le jardin à la française doit beaucoup au jardin italien auquel il a donné plus de prestige ? À propos de jardins, il est intéressant de voir aussi comment Marie-Antoinette, à Versailles, a décidé de créer un jardin très proche du jardin anglais. Elle était autrichienne, n'est-ce pas, et avait ce goût « anglais » qu'elle a donné à la maison dite de la Reine. On voit parfaitement apparaître ici la part de l'autre.

- Dominique Machabert. II n'y a pas d'identité qui ne soit poreuse.

- Álvaro Siza. Absolument. Alors que le monde de l'architecture s'interroge et doute à propos de son devenir, certains architectes comme Jean Nouvel ou Dominique Perrault éprouvent le besoin impérieux d'aller voir ailleurs, de chercher autre chose. Regardez le Louvre Abu Dhabi, capable de déployer des possibilités inouïes qui vont jusqu'à pousser très loin l'exigence que réclame un bâtiment pareil. On y voit très clairement la recherche et l'expression propres à Jean Nouvel. Comme chez Frank Gehry. On ne peut parler chez eux d'une «ligne ». Chacun de leurs bâtiments prend valeur d'exception. Pour les architectes, les opportunités de sortir de la norme sont désormais très rares. C'est pourquoi il convient de saluer ceux qui y parviennent et savent créer de telles conditions.

- Dominique Machabert. Revenons à votre travail et à ces qualités que vous prêtez aux autres. Après Berlin et Paris, parlons de la fondation Iberê Camargo à Porto Alegre au Brésil (1998-2008). Pour justifier de la forme du bâtiment et de son implantation (fig. 3a-b), vous parlez de la parcelle très étroite, de la voie de circulation qui passe devant, de la paroi rocheuse, de la présence du fleuve, le Guaíba...

- Álvaro Siza. Un grand fleuve. C'est un delta...

- Dominique Machabert. Ce sont ces conditions-là qui font décoller les formes comme des bras jetés en avant? 

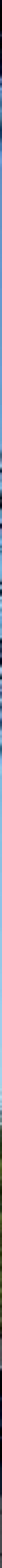


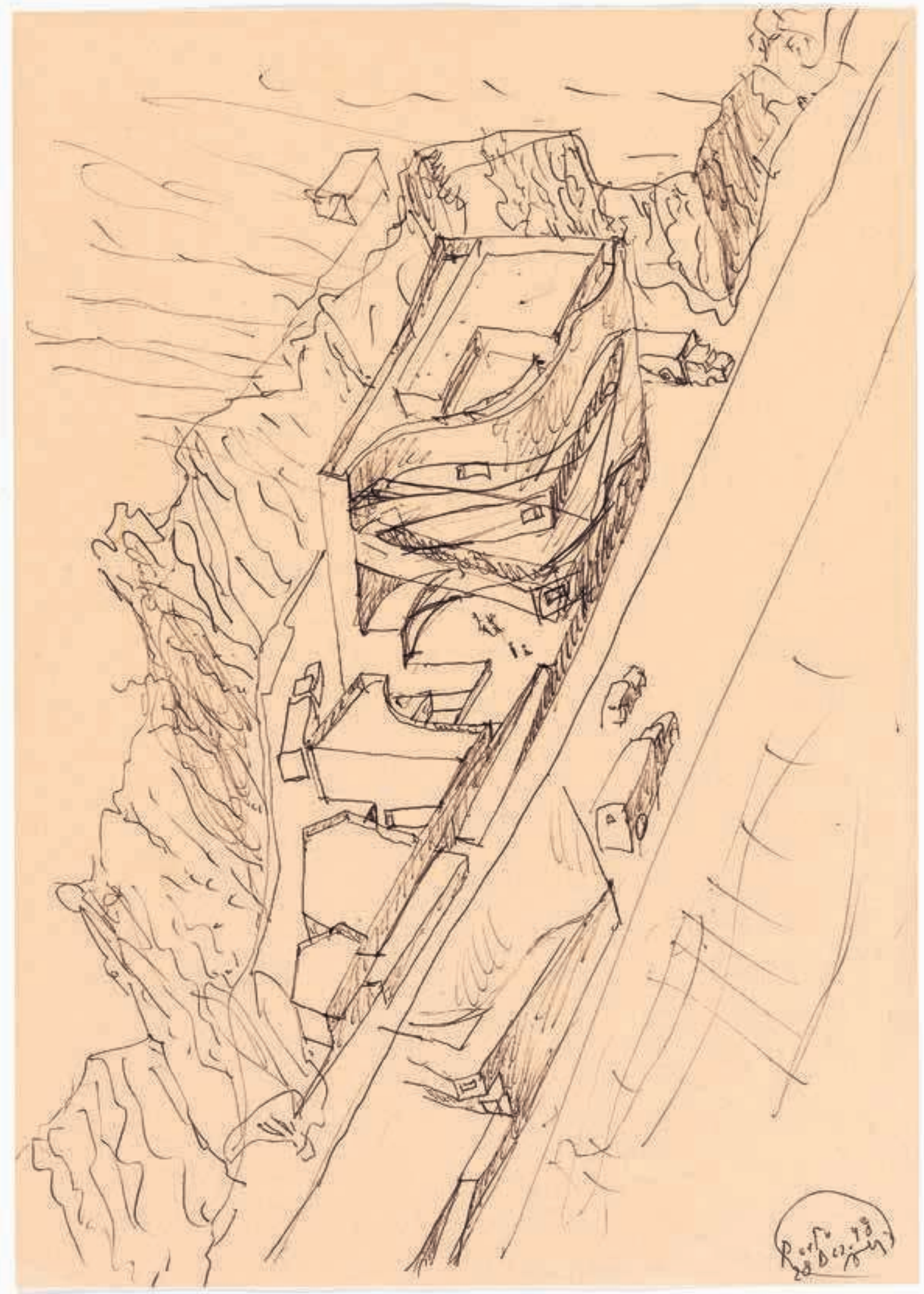


3a-b. Álvaro Siza, fondation lberê Camargo, Porto Alegre, 1998-2008, [a] vue du musée, photographie de Dominique Machabert ; [b] croquis en perspective aérienne, encre sur papier, 29,7 × $21 \mathrm{~cm}$, New York, The Museum of Modern Art, Gift of Jorge Gerdau Johannpeter, inv. $n^{\circ}$ 887.2012 .

- Álvaro Siza. Des bras qui se projettent au-devant du grand espace autour d'un tout petit noyau qui a besoin... de s'étendre [il fait un mouvement ample avec ses bras en aspirant l'air autour de lui]. Vous parlez de la paroi rocheuse. La différence de niveau est assez violente il est vrai. Pourtant la végétation qui couvre abondamment la paroi adoucit ce surplomb important. Pour ce projet, il convenait d'abord de ne pas toucher au rocher alors que la tentation de se coller à lui était grande puisque cela nous aurait fait gagner de la place.

- Dominique Machabert. Au cours d'un précédent entretien à ce sujet, vous parliez aussi de l'ambiance, de l'atmosphère générale qui pouvaient expliquer l'expression d'un modernisme plus généreux au Brésil qu'ailleurs. Est-ce que " l'autre » serait ce modernisme libéré, une architecture plus libérée?

- Álvaro Siza. Oui ! Je suis d'accord pour l'architecture mais dans son paysage. Je pense à Rio de Janeiro où l'on est immédiatement happé par le caractère sculptural du site. On note cette unité qui diffuse un pouvoir énorme : l'immensité de la mer et les rochers comme des sculptures posées dessus.

- Dominique Machabert. Comment un pays, ici le Brésil, en vient-il à participer au projet, à lui donner un caractère, comme ces formes affranchies que votre bâtiment déploie ?

- Álvaro Siza. L'influence de l'architecture moderne brésilienne au Portugal fut immense à partir des années 1950. Je me souviens que Fernando Távora arriva un jour avec un très bel ouvrage, Brazil Builds ${ }^{9}$, qui nous a tous ébahis. Il y avait à l'intérieur un monde nouveau avec des bâtiments, des maquettes, des photos, un graphisme formidable (fig. 4). C'était comme une bouffée d'air. Il y avait d'abord eu l'exposition au Museum of Modern Art de New York, consacrée à l'architecture brésilienne et qui montrait les premières œuvres d'un très jeune architecte, peu connu encore même au Brésil, un certain Oscar Niemeyer invité plus tard à participer à la conception du siège des Nations Unies à New York. Les revues comme Architecture aujourd'hui accordaient une large part à ce qui se passait au Brésil dans chacun de leur numéro. J'avais donc, à propos du pays, un bagage énorme avant même de m'y rendre. Il y avait aussi ce que me racontait mon père qui était né là-bas, à Pará do Belém ; et qui était très jeune lorsqu'il est rentré du Brésil avec sa famille. Nous n'étions encore que des enfants et il nous racontait des histoires d'animaux, de plantes et d'arbres et de bien d'autres choses extraordinaires. À l'entendre, c'était presque un mythe ; quand j'en parle cela remonte de l'enfance et il est resté très présent en moi, et sûrement très influent ensuite, ce merveilleux Brésil, symbole d'un monde nouveau. Vous imaginez ce qu'a pu être mon arrivée sur place pour y travailler. J'avais en tête aussi le travail de Le Corbusier, mes années de formation... Devant l'immense delta, depuis la parcelle minuscule où nous devions construire, au pied d'une paroi rocheuse couverte d'une végétation luxuriante, j'avais le paysage en tête et ce que mon père me disait du paysage. C'était énorme.

- Dominique Machabert. Difficile de distinguer toutes les influences qui peuvent expliquer les choses que l'on fait... 
- Álvaro Siza. Il y a l'inconscient. Quand j'étais là-bas, je ne pensais pas exactement aux récits de mon père. Mais j'avais en moi l'émerveillement de l'enfance. Beaucoup de choses vivent en nous sans que nous sachions toujours ce qu'elles sont et comment elles se présentent. Tant de choses nous traversent qu'il n'est pas possible qu'elles soient toutes conscientes.

- Dominique Machabert. On a l'idée que cette pensée plus ou moins enfouie vous accompagne, que vous lui faites une place.

- Álvaro Siza. Ce n'est pas volontaire. Mais il m’arrive d'avoir l'idée du travail inconscient. Ce sont les choses, parfois ce sont les gens qui, à propos de l'un de mes bâtiments, voient un emprunt, une référence. Je le découvre alors voyant la pertinence, le fondement de la remarque. Mais il est vrai que l'on ne se pose pas ce genre de question quand on travaille.

C'est arrivé avec la fondation lberê Camargo où certains ont vu un emprunt au Serviço social do comércio Pompéia, à São Paulo, de Lina Bo Bardi (1977-1986 ; fig. 5) auquel je n'ai jamais pensé concernant mon bâtiment, du moins consciemment. Et quand on m'en a parlé, alors évidemment... Mais peu de temps avant j'avais vu l'usine Van Nelle à Rotterdam (1925-1931 ; fig. 6), un bâtiment des années 1930. Il y a des passerelles aussi, les mêmes que chez Lina Bo Bardi. Alors on peut logiquement penser qu'elle aussi connaissait ce bâtiment comme moi, comme d'autres auteurs de bâtiments avec des passerelles. En ce qui me concerne, ce n'est que plus tard que j'ai fait le rapprochement.

Des expériences glanées au cours d'une vie et mêlées les unes aux autres vont jusqu'à former quelque chose dont on ne saurait dire à qui, à quoi elle appartient. De cela on tire des décisions dont on identifie l'origine ou pas. Mais sait-on toujours ce que l'on fait?

4. Oscar Niemeyer, la maison de l'architecte, 1942. Double page de Brazil Builds. Architecture New and Old/ Construçao brasileira. Arquitetura moderna e antigua, 1652-1942, New York, The Museum of Modern Art, 1943, p. 166-167.

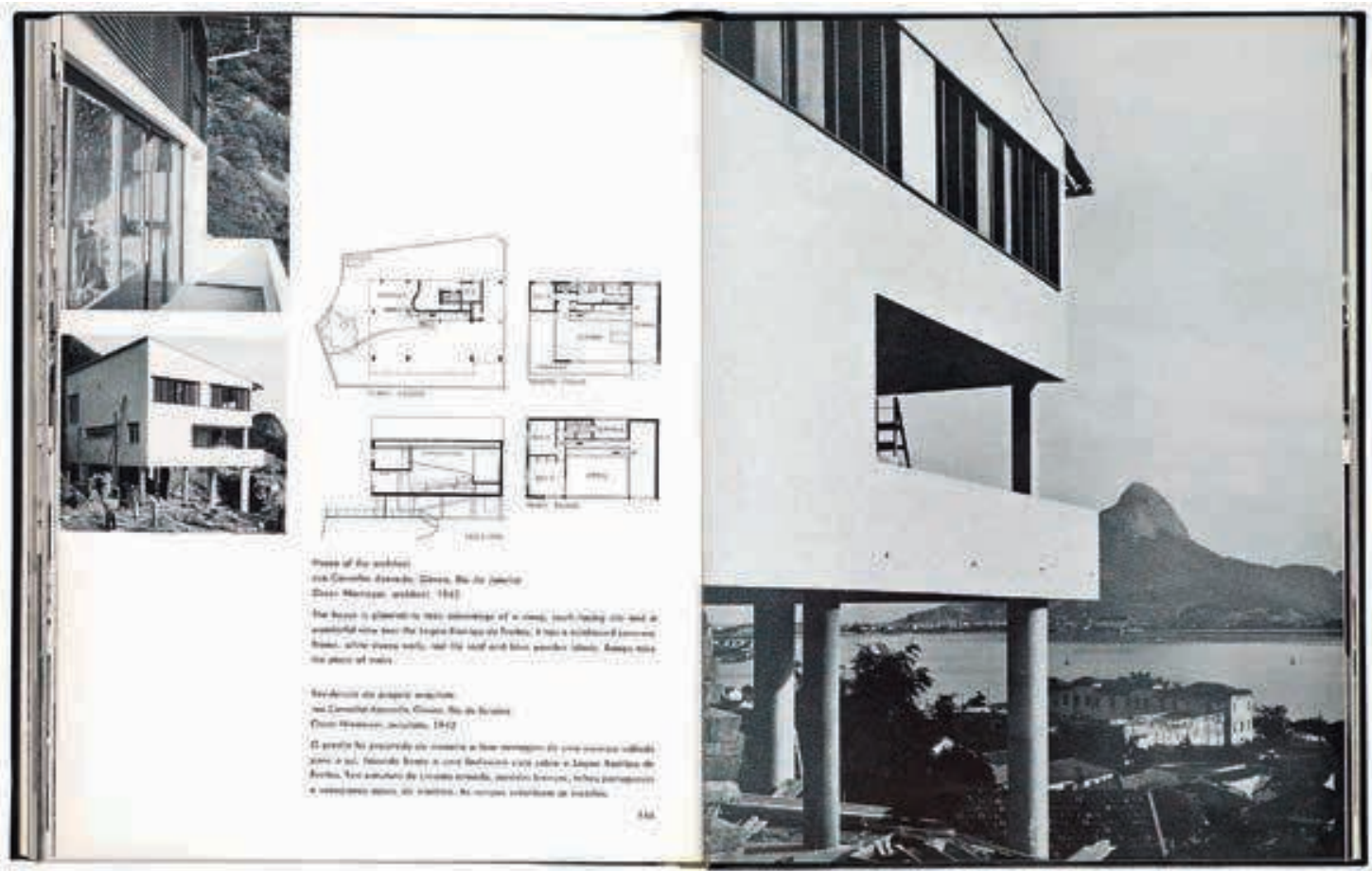




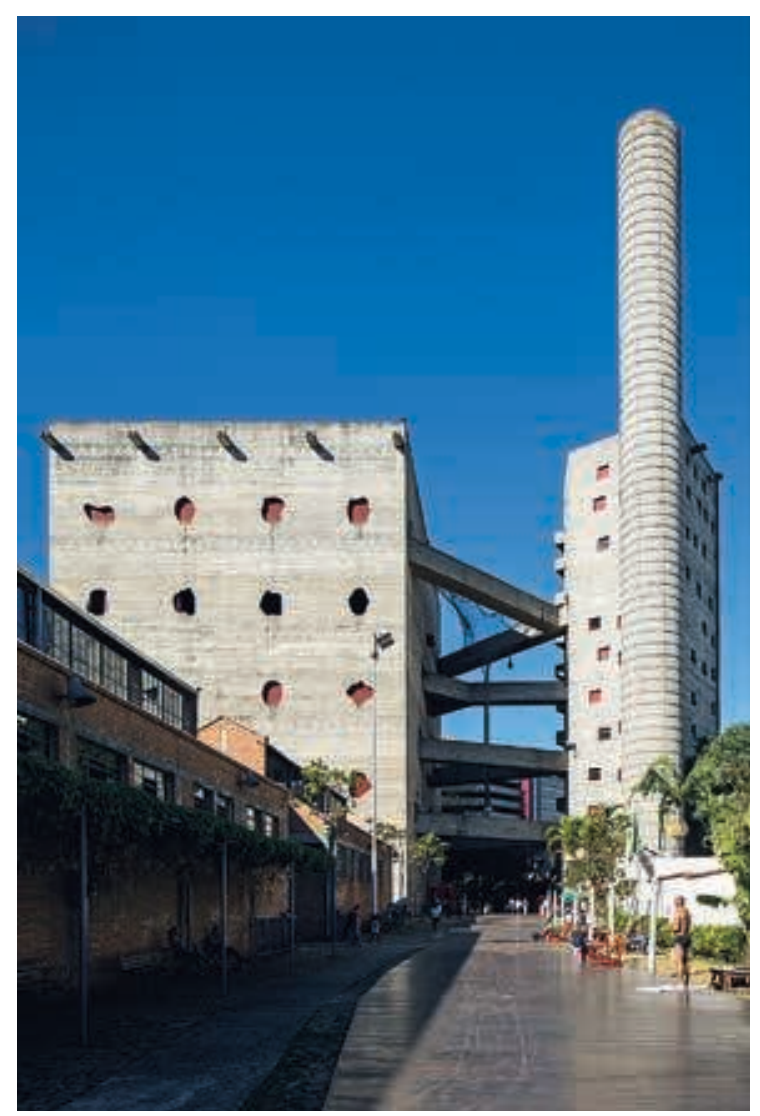

5. Lina Bo Bardi, Serviço social do comércio Pompéia, São Paulo, 1977-1986, photographie de Leonardo Finotti.
- Dominique Machabert. À propos de vos logements collectifs à La Haye (Punt en Komma, Suze Robertsonstraat, 1986-1989), vous avez parlé du haagse portiek. Il s'agit d'une typologie traditionnelle un peu oubliée, aux Pays-Bas, et qui permet à chaque appartement d'avoir un accès partagé avec l'extérieur et avec la rue. En exhumant le haagse portiek (fig. 7a-b) vous portiez, sans le vouloir, le débat sur la place publique et jusqu'au Parlement. Les uns étaient pour, les autres contre.

- Álvaro Siza. Il ne s'agissait pas d'un si grand conflit. Mais il est vrai que ma proposition avait été refusée au niveau municipal, pour des raisons de sécurité, disait-on. La discussion eut donc lieu au niveau du Parlement qui adopta, grâce aux voix de la droite, une proposition venue - comble de l'ironie - d'un type de gauche. Cette histoire remonte au tout début du projet, quand la maîtrise d'ouvrage m'invita à faire un grand tour en bus pour m'indiquer, in situ, ce dont ils ne voulaient pas. À Rotterdam, ils m'ont montré ce fameux système de galerie très présent dans la ville mais qu'ils estimaient dangereux et peu sûr. Ils préféraient, pour chaque appartement, une entrée strictement individuelle et fermée. Mais avec son système de portique clairement ouvert, visible et rehaussé jusqu'au premier étage qui permettait pour chaque appartement un accès direct et indépendant, le haagse portiek, relégué aux antiquités pourtant, avait fait son chemin en moi.

- Dominique Machabert. «L'autre » c'est aussi l'autre de la tradition loin d'être éteinte?

- Álvaro Siza. C'était l'un des thèmes majeurs de Távora. Quand il écrit « Le problème de la maison portugaise » C'est ce point-là qu'il éclaire, sur le bon et le mauvais usage, quand il est rétrograde, de la tradition.

\section{- Dominique Machabert. Vous sentez-vous plus disponible à l'étranger qu'au Portugal ?}

- Álvaro Siza. Pas davantage. Longtemps je n'ai connu Évora (fig. 8) que très superficiellement par exemple. Je n'y avais jamais résidé plusieurs jours d'affilée, ni fait de séjours répétés, ignorant alors tout sur la façon qu'ont les choses, l'architecture, les gens de se transformer, de bouger au gré des jours, des saisons, du climat - ce qui s'appelle connaître une ville. Et il faut noter qu'au Portugal il y a une grande différence physique et même culturelle entre le Nord et le Sud. Je ne fais donc pas de différence fondamentale entre l'étranger et le Portugal.

II n'empêche qu'il y eut des voyages à l'étranger, à l'occasion de vacances surtout, qui m'ont fait grande impression. Je pense à mon premier voyage au Maroc baigné dans une ambiance que je n'ai jamais oubliée. C'était à la tombée du jour, dans une maison de thé, 


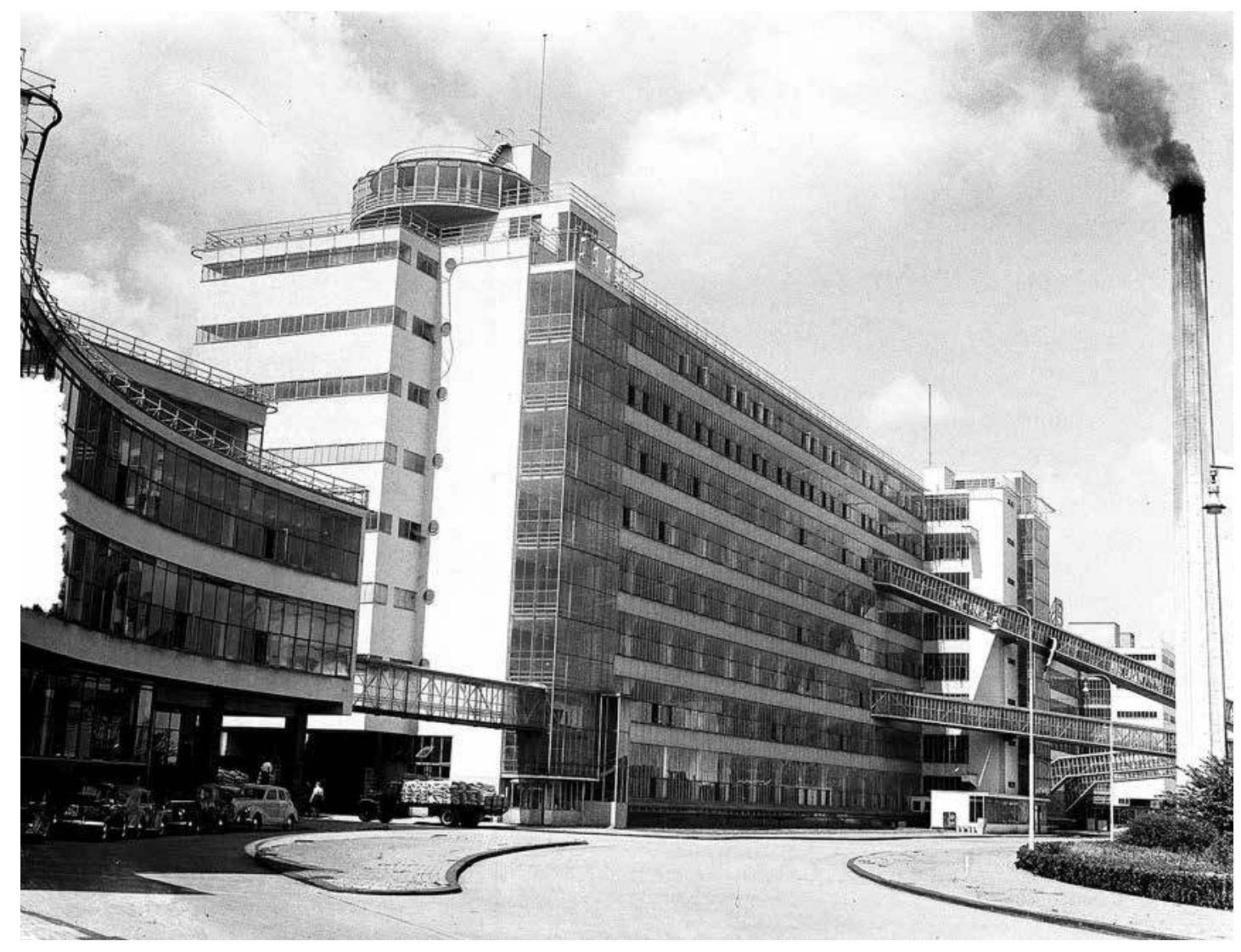

à l'air libre, dans le soleil couchant, au moment de l'appel à la prière. Mais cette qualité d'atmosphère, je I'ai éprouvée aussi dans le massif de Gerês, au Nord 6. Leendert van der Vlugt et Johannes Brinkman, usine Van Nelle, Rotterdam, 1925-1931, photographie de Carel L. de Vogel, La Haye, archives d'État des Pays-Bas.

du Portugal. Nous avions passé trois jours sur place et avions dormi dans un de ces abris en pierre qui servent aux bergers pendant les périodes d'estive. C'était au moment de l'« Enquête", j'étais en compagnie de quelques amis qui voulaient voir les palheiros, sorte de petits greniers en pierre, très beaux.

- Dominique Machabert. Vous semblez insister sur le caractère non professionnel de vos voyages « mémorables».

- Álvaro Siza. J'ai écrit un texte où je parle de ces voyages fait sans pression, sans raison particulière $^{10}$, comme à l'occasion de ce séjour à Rome, où je dis le goût que j'ai eu à me perdre dans les rues, meilleur moyen selon moi pour expérimenter les choses, les apprendre, et pour capter l'atmosphère, poussé par rien d'autre que par le désir de découvrir sans se sentir obligé d'aller voir le Panthéon par exemple. La rencontre, le hasard.

- Dominique Machabert. Vous parlez souvent d'atmosphère, d'ambiance, d'en « capter » quelque chose, la valeur de l'instant où toute la complexité semble se tenir. Concernant les projets d'architecture, dans des situations plus contraignantes, parvenez-vous toujours à trouver cette disposition propice à la rencontre, à l'étonnement ? 
- Álvaro Siza. Il n'y a pas de méthode, pas de calcul. Mais il est vrai que j'ai pris conscience peu à peu que le caractère plus aléatoire de la découverte auquel je me prête à mon insu, dirai-je, a finalement autant d'importance que la prise en compte volontaire et indispensable des objectifs que nous ne saurions négliger. Je ne saurais donner d'exemple précis mais disons que le contexte d'un projet conduit à forcer la découverte, à en ôter un peu le plaisir. Le plaisir est indispensable en architecture. Et sûrement faut-il repérer là une certaine perte. Une perte dans notre capacité à absorber profondément, sans intention

7a. Álvaro Siza, axonométrie du haagse portiek, montrant la conception des escaliers qui mènent à chacun des appartements, pour l'immeuble d'habitation Punt en Komma (La Haye, 1986-1989), 1985, stylo rouge et noir sur film, Montréal, collection Centre canadien d'architecture, fonds Álvaro Siza, dossier 178-268-02, réf. ARCH274291 (don d'Álvaro Siza). particulière, ce qui ne vient pas de l'avoir obtenu à force d'effort et de volonté, quelque chose qui vient et s'enregistre tout seul.

- Dominique Machabert. Veiller à faire avec le caractère moins assuré, plus incertain, plus diffus des choses et à laisser la rencontre, la surprise opérer, est-ce que
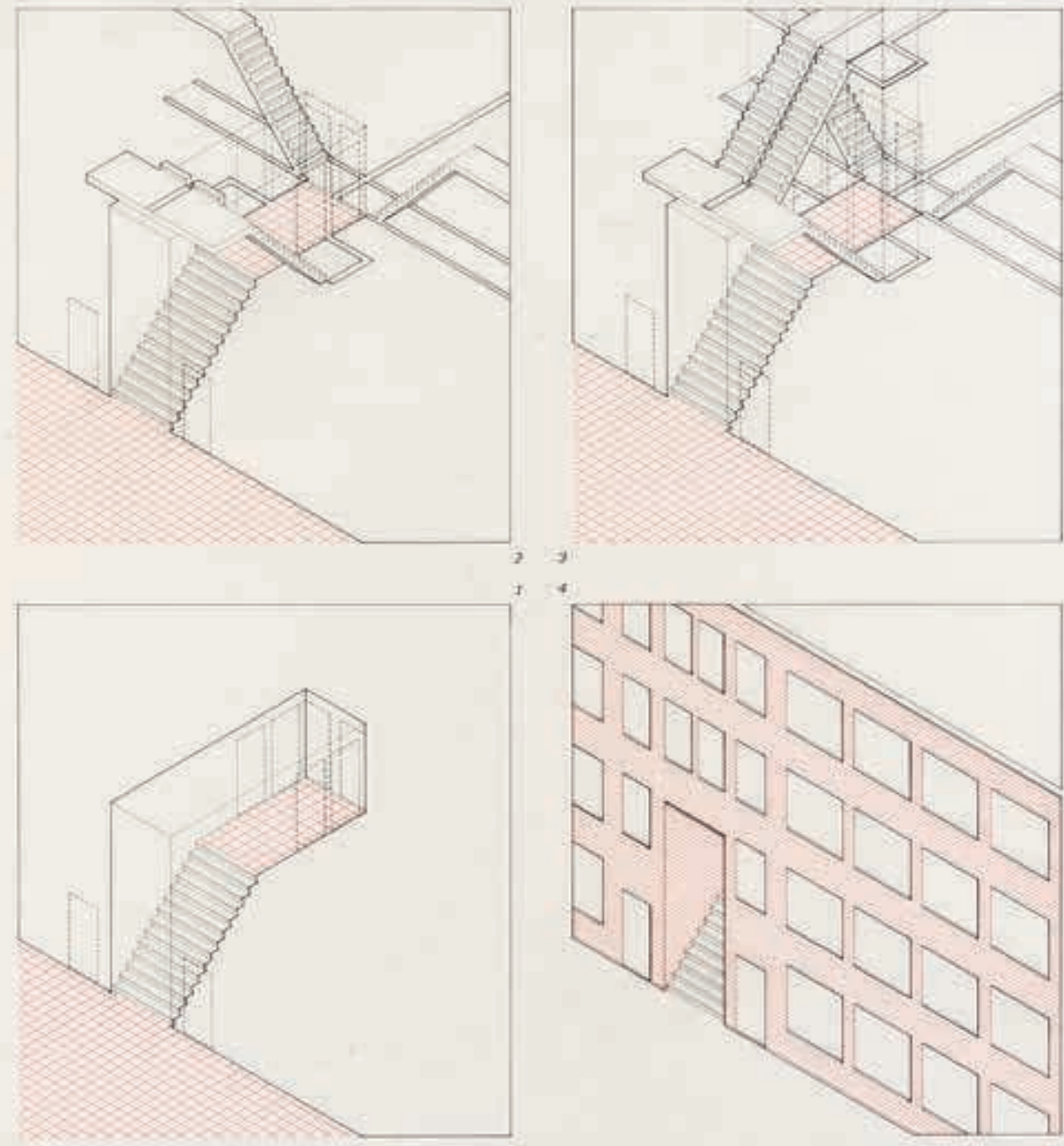


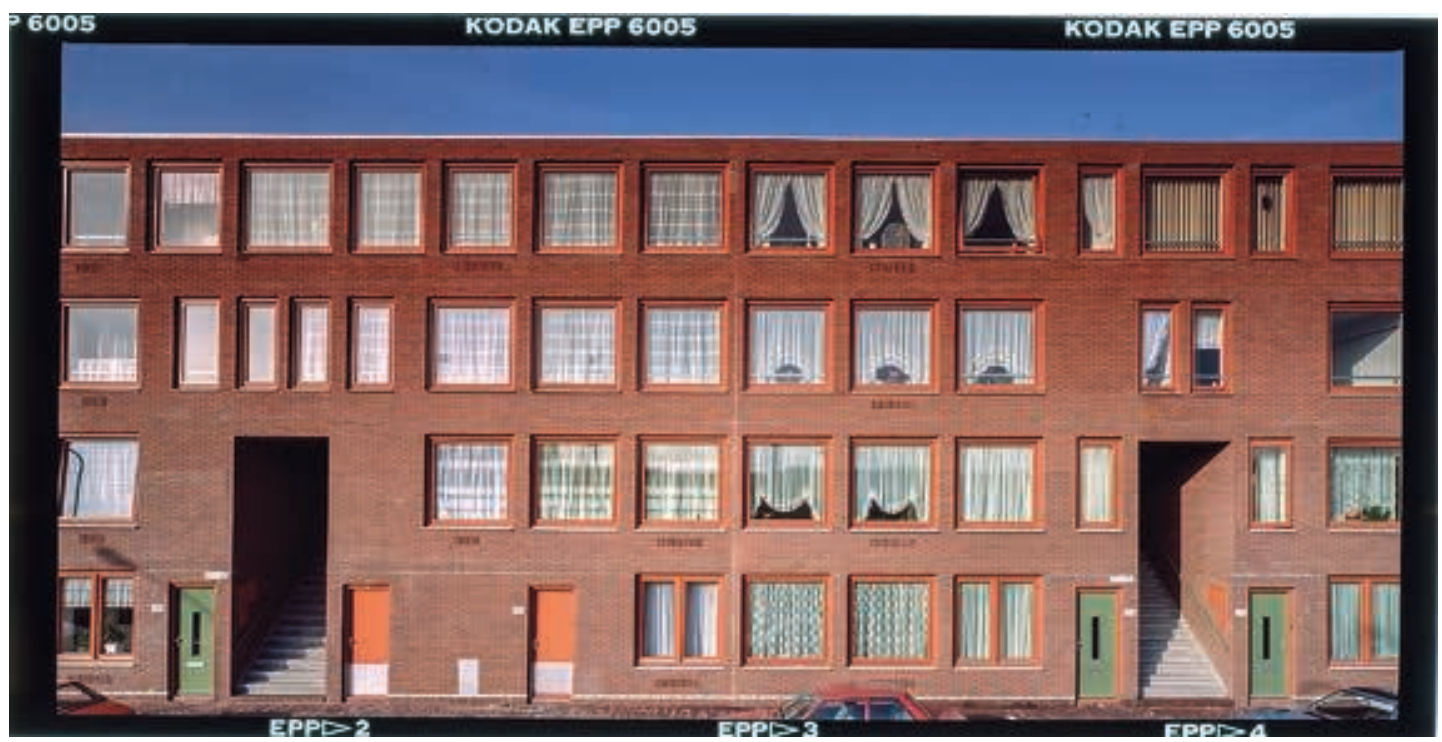

cela suppose un risque plus grand, peut-être une plus grande angoisse? 7b. Álvaro Siza, immeuble d'habitation Punt en Komma, La Haye, 1986-1988, photographie de Peter de Ruig.

- Álvaro Siza. Un risque sûrement. Mais ce n'est pas un truc. Prendre des risques n'est pas un truc mais un désir spontané de s'ouvrir. Au lieu d'aborder le projet en m'assurant d'avoir tout en main, toutes les données, toutes les informations, en bouclant tout, je me prête plutôt à ce qui advient pour ensuite, et ensuite seulement, l'analyser. Trouver en soi une disposition, une souplesse d'esprit. Tenter d'avancer sans crainte, sans lassitude, pour être le plus présent possible à la situation, n'être pas anxieux et accepter d'être ignorant d'une quantité de choses sachant qu'elles viendront à s'éclairer. Dans une certaine mesure, la fatigue conduit à trouver cet état.

- Dominique Machabert. Cela réclame une grande confiance en soi.

- Álvaro Siza. Plus que de confiance je parlerais d'abandon, de se laisser aller à une sorte d'abandon au lieu de forcer, d'étouffer la chose plaisante par souci d'efficacité. Une sorte de spontanéité, d'accueil... Quand je démarre un travail, devant l'immensité de la tâche qui se présente à moi, devant les différentes approches et disciplines en jeu qu'il faudra aborder, sachant déjà qu'il faudra tout étudier point par point, je suis gagné par une peur qui me submerge. C'est d'abord sous ce jour que les choses se présentent; un blocage presque.

Alors devant cela, plutôt que de prendre des précautions excessives qui maintiennent, dès le départ, le projet sous la contrainte, je fais le pari d'une sorte d'abandon pour qu'une certaine fraîcheur anime le travail délivré de l'anxiété qui paralyse. C'est comme un remède.

- Dominique Machabert. Est-ce cela la création?

- Álvaro Siza. Oui. Et c'est vrai pour d'autres domaines. Picasso disait avoir consacré toute sa vie à réapprendre à dessiner comme les enfants. C'est un peu la même chose que ce que nous disons : tenter de reconquérir une spontanéité naturelle perdue. 


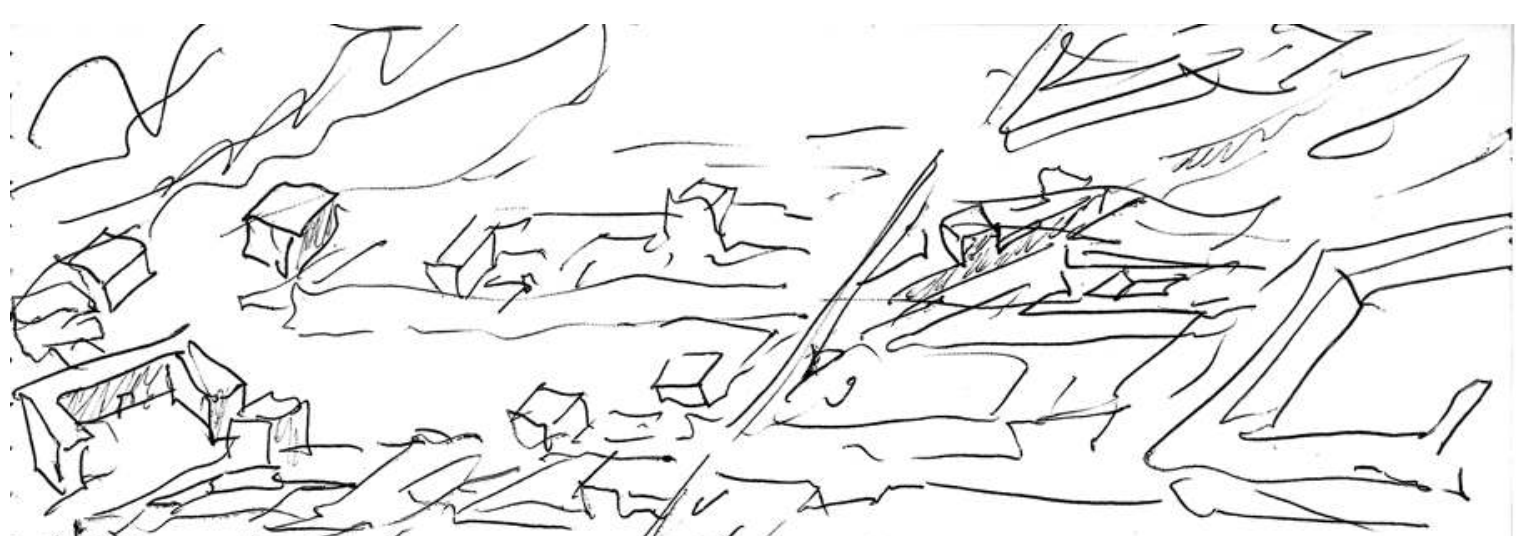
或-

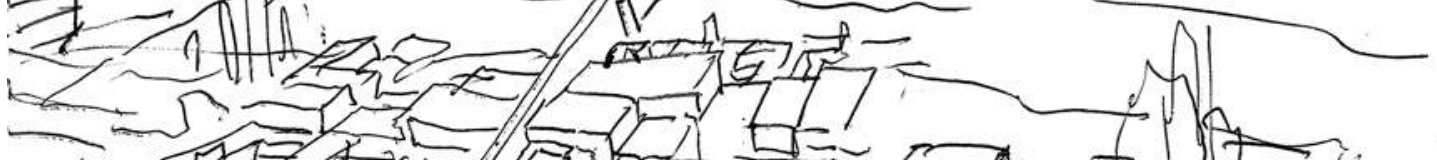

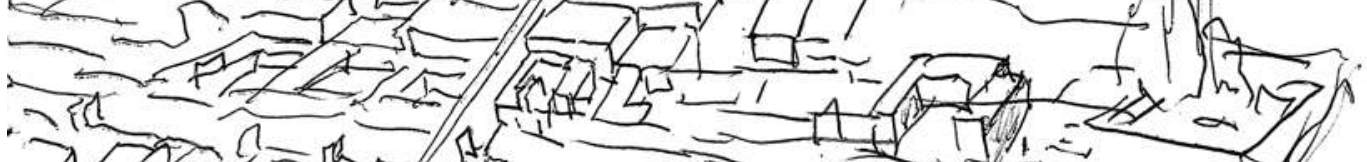

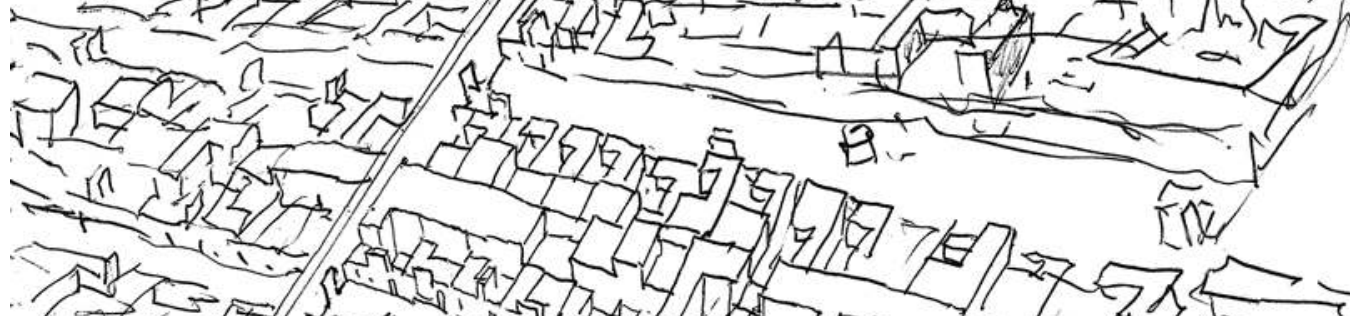
㞸 - 5 .

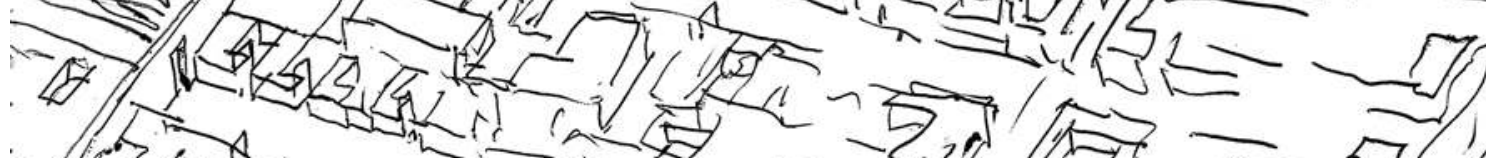
- 万人

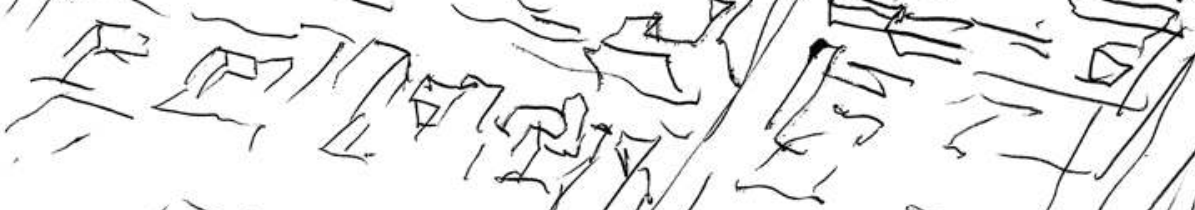

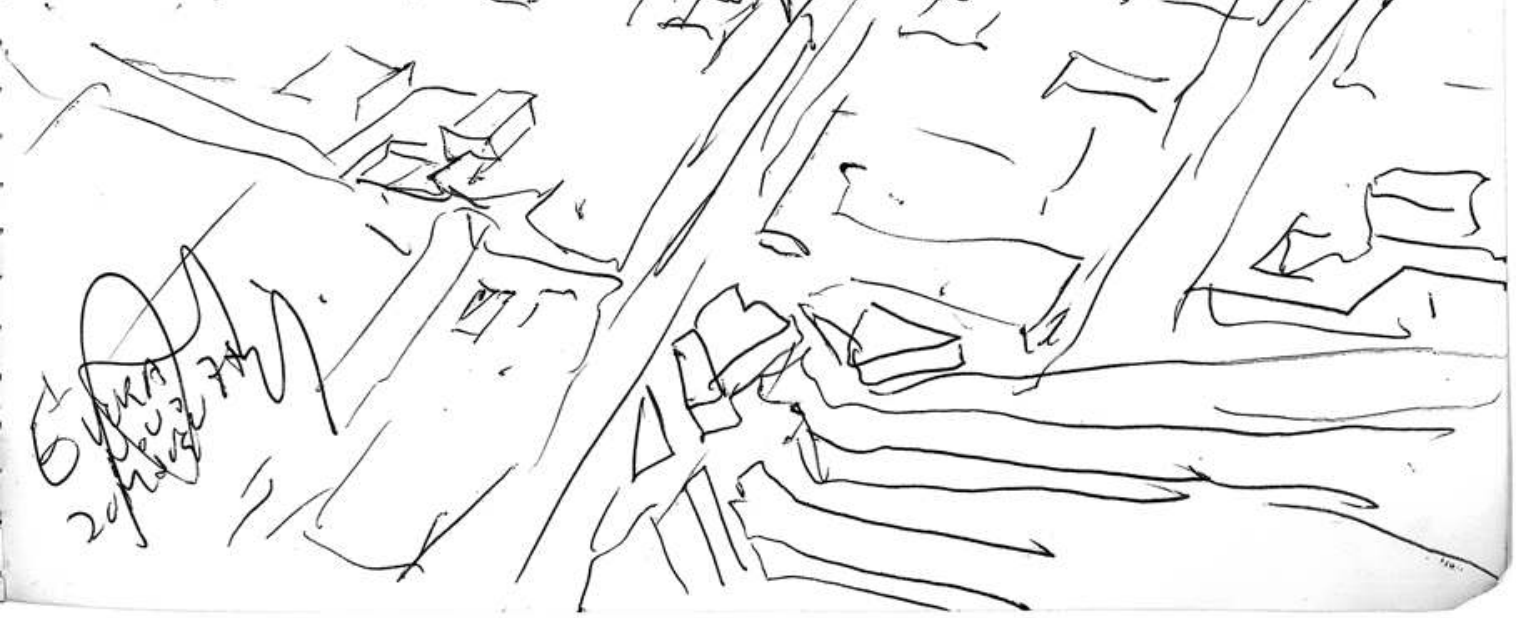


- Dominique Machabert. Comment témoigneriez-vous de cette reconquête pour vous?

- Álvaro Siza. La rencontre. Je me souviens d'un écrivain qui racontait qu'à un certain moment le personnage de son roman lui échappait pour mener sa vie propre. Comme le développement du roman mû par son personnage, on doit aussi, dans le projet d'architecture, laisser venir les rencontres, laisser les expériences, les références advenir sans exercer sur elles trop de frein, trop de pression. Laisser vivre le projet comme le personnage d'un roman.

Mais à le laisser vivre trop sans lui opposer de limite, il peut s'avérer monstrueux. II faut alors le contrôler mais sans l'asphyxier. Il s'agit donc d'un balancement entre raison, rationalité et mouvement instinctif, intuitif, d'une vigilance qui n'empêche pas une sorte d'insouciance.

- Dominique Machabert. Avec l'expérience, est-ce plus aisé pour vous aujourd'hui qu'hier?

- Álvaro Siza. C'est plus difficile aujourd'hui à cause des contraintes extérieures de plus en plus pressantes, plus difficile en raison de la forme que prend le travail, dont l'accès est toujours plus appareillé. On prétend que l'ordinateur ouvre à plus de rapidité et à plus d'efficacité. C'est sans compter avec le bougé constant, subtil, auquel un projet a affaire ; un bougé qui ne procède pas d'une marche forcée qu'ordonnerait un logiciel réglé pour sortir du projet, toujours le même en quelque sorte.

L'informatique, l'ordinateur sont des outils indispensables aujourd'hui, même dans le domaine créatif. L'usage qu'on en fait à l'agence facilite des opérations et permet de comparer de façon efficace des solutions alternatives au projet. Pourtant, le risque serait de croire qu'ils peuvent se substituer à la recherche personnelle et que tout est déjà là, dedans, qu'il suffit de cliquer pour l'obtenir. C'est que l'usage de tels outils réclame d'être mesuré et gardé sous contrôle. En effet, en s'isolant dès le début devant sa machine sans rien voir autour, on se prive d'une ouverture immense et de l'apport incomparable du travail à plusieurs (fig. 9).

- Dominique Machabert. On sait que le dessin est essentiel pour vous, qu'il est votre outil de recherche privilégié, de dialogue même...

- Álvaro Siza. Privilégié parce qu'il produit chez moi une émotion (fig. 10). Et pour parler bien franc et au-delà de sa performance, l'outil informatique ne provoque chez moi aucune surprise.

- Dominique Machabert. Frank Gehry, que vous appréciez autant que son travail, s'appuie beaucoup sur l'outil numérique.

- Álvaro Siza. J'ai travaillé avec lui pour un projet à Los Angeles qui n'a finalement pas été réalisé. M'étant rendu sur place plusieurs fois je l'ai vu faire, comme au cours de cette fameuse séance où cherchant à produire quelque chose, de façon tout à fait indisciplinée et avec une joie immense, il lança au beau milieu de la maquette un bout de polystyrène. C'est alors que les logiciels travaillent à absorber cet événement qui, d'aléatoire, devient une forme. Je ne sais si Gehry procède toujours ainsi ou si ça n'était qu'un mouvement passager,

8. Álvaro Siza, logements sociaux du quartier de la Malagueira (1 200 logements/17 hectares), Évora, 1973-1977. mais je l'ai bien vu faire. Et comme je connais ses dessins et ses lignes qu'il pousse de façon assez instinctive, je peux témoigner de l'authenticité de sa recherche. Ce qui semble 


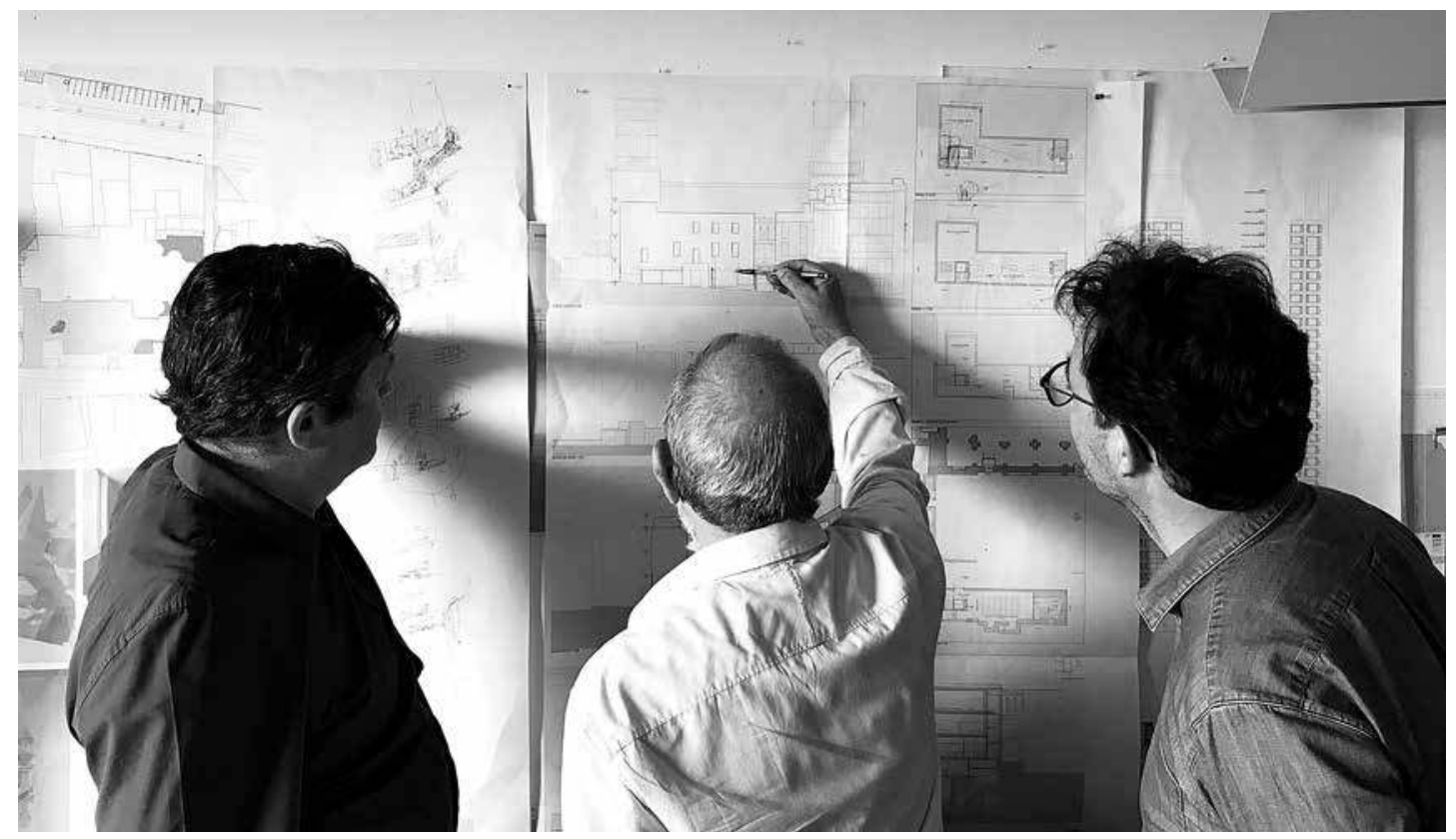

9. Álvaro Siza au travail avec ses collaborateurs. Photographie de Dominique Machabert

venir d'instinct ou ce qu'on appelle encore le geste spontané met bien souvent du temps à advenir. Il vient de loin, il est le fruit d'une recherche immense et d'une quantité de croisements, de rencontres.

Je me souviens d'un très beau texte, très court aussi, d'Alvar Aalto. Devant la difficulté qui pouvait l'empêcher d'avancer dans son travail, il raconte qu'il décidait de tout suspendre et de dessiner ou de peindre tout autre chose. Alors et contre toute attente, la difficulté se levait et le travail pouvait reprendre son cours ${ }^{11}$.

\section{- Dominique Machabert. Est-ce que cela fonctionne aussi pour vous?}

- Álvaro Siza. Oui, mais surtout à l'époque où je travaillais seul ou presque, avec seulement deux ou trois collaborateurs. Désormais la recherche est moins soumise à une production directe d'architecture. On travaille avec des gens d'horizons différents, avec leur méthode, leurs exigences propres. II y a plus d'inertie. Je peux travailler par exemple à une certaine qualité d'espace pour un projet donné. Je vais donc faire des esquisses dont certaines ne serviront à rien, mais je les fais quand même, explorant ainsi des alternatives que je demande à mes collaborateurs d'intégrer, de sorte que cela bouge, un détail... À un certain moment, je me fixe sur un point que je pousse pour voir où cela nous conduit, parfois vers une impasse. Mais cela n'avance pas de façon aussi directe qu'avant.

- Dominique Machabert. Vous parliez d'une époque antérieure où vous travailliez plutôt seul. Est-ce que les moments de solitude sont devenus plus rares?

- Álvaro Siza. Il y a toujours des moments de solitude mais ils sont moins indispensables qu'avant. Désormais les projets sont plus souvent soumis à une pression qui exige de nous d'être plus efficaces, plus rapides et donc plus systématiques. Alors il y a moins de solitude, 
disons qu'il y a moins de perte de temps, ce qui réduit la recherche, le travail d'investigation et donc les propositions. Bien souvent, un projet procède par bonds successifs au fil d'un travail critique dont il est sans cesse l'objet. À l'inverse de ce procédé-là, la normalisation systématique conduit plutôt à une censure qui barre l'accès, une à une, à chacune des voies potentielles qui restent inexplorées.

- Dominique Machabert. Je pense à Fernando Pessoa seul mais investi, mû par ses hétéronymes.

- Álvaro Siza. Je ne me sens pas investi de la sorte, même si je crois que les circonstances, les rencontres, le contexte, l'atmosphère et bien d'autres choses encore nous bougent, nous poussent à faire des choix, difficiles parfois, éprouvants même. C'est cela la création sans doute, jamais toute droite mais ouverte, constamment ouverte, ce qui oblige alors à savoir synthétiser, à savoir se concentrer intensément et à pouvoir contrôler. Ce que l'on peut considérer comme soi-même n'est certainement pas étranger à ce qui se passe en dehors, autour de soi : une rencontre, un échange, un conflit, le contact des choses extérieures à nous-mêmes qui s'en mêlent...

- Dominique Machabert. Jorge Luis Borges dit quelque part ceci : "C'est à l'autre, c'est à Borges que les choses arrivent. Moi je marche dans Buenos Aires ${ }^{12}$. " Est-ce qu'il vous arrive, à vous aussi, de vous dire que c'est à «l'autre ", à Siza, que les choses arrivent?

- Álvaro Siza. C'est la forme inattendue de soi. De mon côté, je ne me vois pas comme une chose fermée, finie. Mon identité varie au gré des situations. Je ne suis pas toujours le même. Et ce n'est pas problématique ni douloureux. Pour moi c'est du dehors, d'une stimulation extérieure que tout à coup un instant très spécial, décisif, déclenche quelque chose.

- Dominique Machabert. Je note chez vous une sorte de retrait qui ne permet pas d'identifier avec certitude votre travail, différent ici et là, et qui vous rend inclassable. C'est plus diffus, plus souple. Eduardo Souto de Moura parle "d'adéquation portugaise». Je proposerais alors ceci vous concernant : que votre inclination à " capter ", à porter les traits de l'autre, à Berlin, à La Haye ou ailleurs viendrait du fait d'être portugais.

- Álvaro Siza. On pourrait dire que la souplesse s'impose aussi, qu'elle est une exigence. Et je n'oppose pas identité et souplesse diffuse, fermeture et ouverture. Les deux vont ensemble dans un continuum. Par ailleurs, je ne me reconnais pas comme un architecte " portugais ». J'ai lu des écrivains originaires d'autres régions du monde où je trouve la même disponibilité, les mêmes attentions, la même ouverture que vous prêtez aux Portugais.
10. Álvaro Siza, dessin à l'encre noire sur une page du carnet 168 [Berlim Elder's Club R.], février 1984, Montréal, collection Centre canadien d'architecture, fonds Álvaro Siza, ARCH281743 (don d'Álvaro Siza).

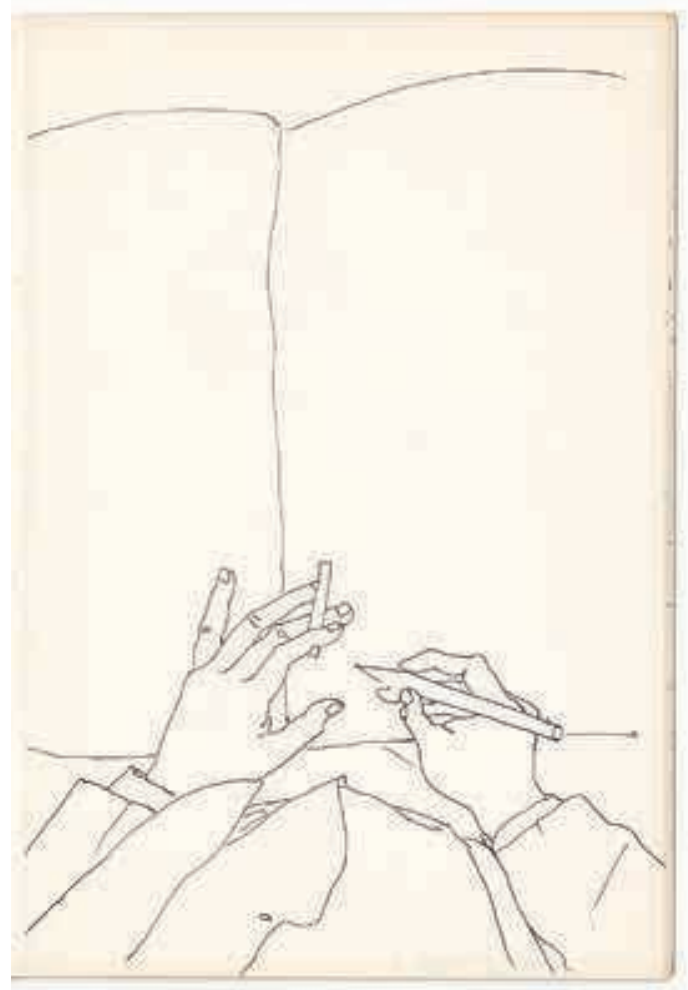




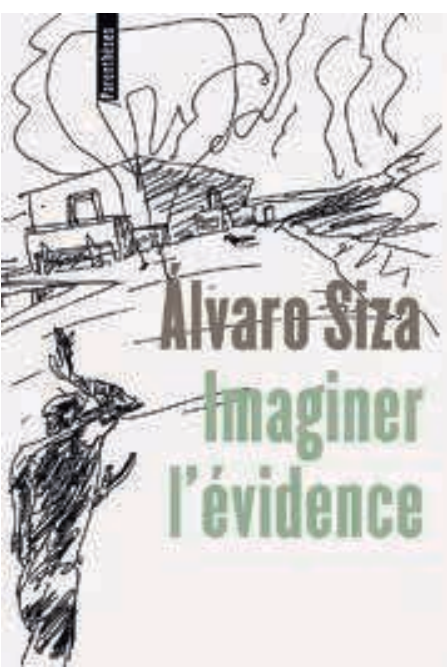

11. Álvaro Siza, Imaginer l'évidence (première de couverture), Marseille, Éditions Parenthèses, 2012).
Il y a quelque chose ici que je voudrais vous montrer [il se lève]. C'est pour une exposition. Ce sont des dessins extraordinaires d'animaux fait par un enfant d'à peu près six ans |il les montre]. En même temps qu'une réelle fermeté du trait, vous remarquez ce sens de l'observation et la captation de ce que c'est. On voit que c'est un tigre, une girafe, tout y est. Mais c'est fait avec une telle liberté ! Et l'on pourrait dire, à quelques exceptions près peut-être, que tous les enfants font des dessins extraordinaires. Et c'est ce que repère si bien Picasso. Avec l'école, la plupart des enfants perdent cette ouverture, cette spontanéité. Je veux parler d'une spontanéité non abstraite, toute concentrée dans le monde qu'ils voient... Quand vous parlez d'identité dynamique, souple, qui échappe à la fixité locale et définitive, je vois dans ces dessins d'enfant un rapport à cela. Alors certes le Portugal est un croisement important d'influences culturelles. Mais il n'est pas le seul. Voyez la Syrie, par exemple, qui est un croisement de cultures immense.

- Dominique Machabert. Dans votre livre Imaginer l'évidence ${ }^{13}$ (fig. 11) vous parlez de Rio de Janeiro, de Macao et de la façon dont les Portugais ont su faire avec les circonstances et les situations locales. À défaut de pouvoir imposer un modèle de ville, ils se sont appuyés sur "l'autre ", sur l'existant : le relief, la géographie... La colonisation portugaise a été, à ce titre, très différente de la colonisation espagnole.

- Álvaro Siza. Différente mais la colonisation portugaise fut aussi très violente.

- Dominique Machabert. Alors éclairons cet autre point : vous avez témoigné de vos difficultés - une sorte d'inhibition avez-vous dit - à l'occasion de vos premières tentatives à travailler l'azulejo, matériau très identitaire au Portugal s'il en est mais qui vous a pourtant intimidé (fig. 12)?

- Álvaro Siza. Il se pourrait qu'un matériau, par sa proximité, présente des difficultés avant qu'il ne devienne agile entre des mains d'abord un peu engourdies. J'admets qu'il puisse être plus difficile pour moi de travailler à Porto qu'à Berlin. Travailler à Porto que j'ai sous les yeux tous les jours, depuis l'enfance, réclame nécessairement une attention supplémentaire. C'est pourquoi les voyages sont fondamentaux pour les architectes. Le travail s'il est créatif exige d'être sollicité, dérangé. Sinon on étouffe.

- Dominique Machabert. Un projet d'architecture serait-il toujours exploration d'autre chose, encore?

- Álvaro Siza. Oui, mais sans les affres de devoir le faire pour ceci ou cela. Explorer sans raison particulière, naturellement. C'est pourquoi il est important pour le projet de veiller à garder une certaine fraîcheur en soi. C'est en portant mon attention sur ce qui ne semble pas directement intéressant mais qui est là, présent, insistant même, que je trouve à me libérer de l'étreinte du projet. Je peux le dire de tous, même s'il est vrai que dans certaines circonstances, comme au Brésil, les conditions réunies pour travailler étaient assez exceptionnelles. Je me suis senti soutenu là-bas, à ma place, requis en tant qu'architecte. Car il arrive, à d'autres moments, qu'on ne soit pas les bienvenus. 
- Dominique Machabert. Votre attention qui s'empare de la moindre manifestation concrète servirait-elle aussi de diversion, en quelque sorte, devant l'étreinte trop grande du projet?

- Álvaro Siza. Oui. Comme je dois à mon goût pour la sculpture mon intérêt plutôt tardif pour l'architecture avec Antoni Gaudí d'abord, puis avec Aalto. Une diversion comme vous dites. Mais les situations de blocage, l'étreinte, proviennent rarement des choses concrètes. Au contraire, en architecture, les difficultés proprement dites, réelles poussent toujours en avant. Je me souviens de deux projets de maison à dix ans d'intervalle, à Póvoa de Varzim dans une banlieue de Porto de qualité médiocre, avec tout autour, dans les deux cas, des bâtiments horribles. Dans le cas du premier projet, il s'agissait d'une maison avec un patio complètement fermé avec, côté rue, juste quelques fenêtres et de grands vitrages côté patio (fig. 13). J'avais même sollicité un paysagiste pour qu'il investisse le patio de façon à créer une sorte de "paradis » au milieu d'une situation bien laide et dont il fallait se défendre, qu'il fallait faire oublier à tout prix, pensai-je. On m'a beaucoup reproché par la suite mon attitude qui consistait à ne rien vouloir voir de ce qui se passait autour. J'ai alors beaucoup réfléchi à mon métier, à sa dimension éthique. Pouvais-je tourner le

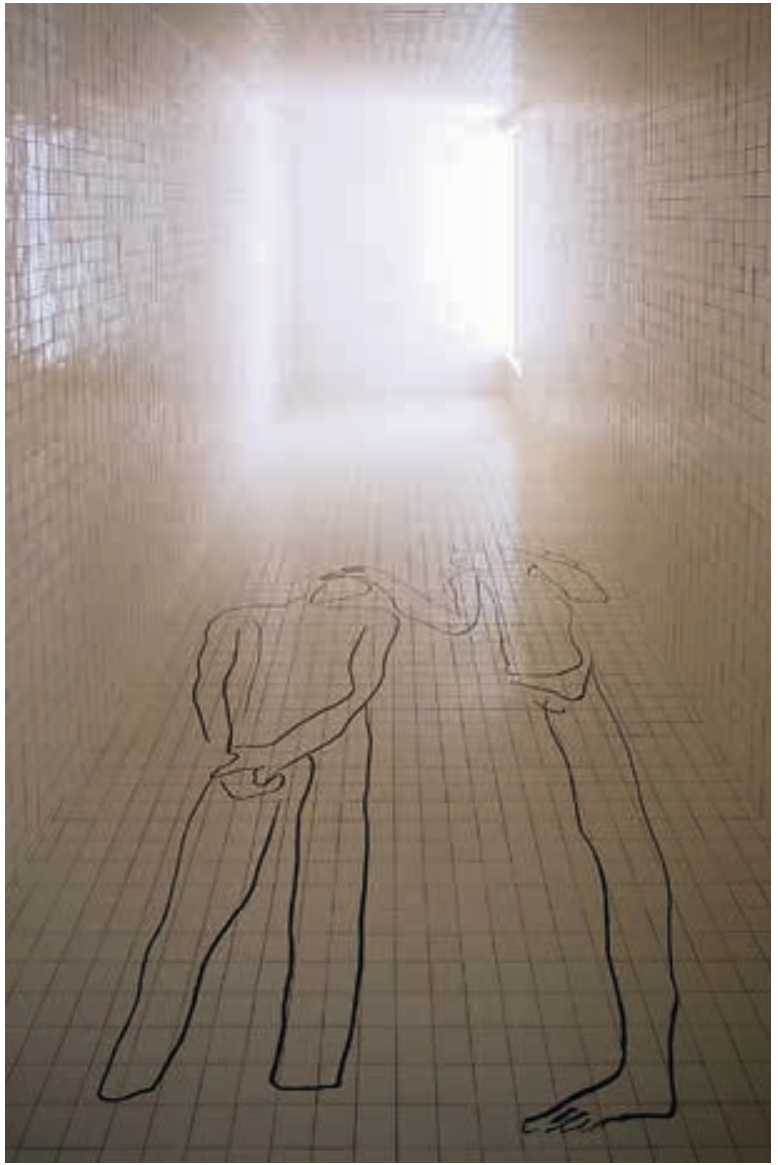

12. Álvaro Siza, Le Baptême du Christ, 2007, dans le baptistère de l'église Sainte-Marie, Marco de Canavezes, photographie de Fernando Guerra. dos à des situations pareilles, alors qu'il en existait une quantité d'autres, partout, problématiques et peu stimulantes, aussi bien d'un point de vue esthétique? Dans ces conditions, il valait mieux changer de métier tout de suite. C'est alors qu'un client qui avait bien aimé la maison à patio faite dix ans plus tôt me commanda la même. Mais la parcelle était bien trop petite pour un projet pareil organisé autour d'un patio. Alors, plutôt que de fermer, j'ai ouvert vers l'extérieur (fig. 14). J'y étais forcé par la situation, certes, mais je voulais aussi en finir avec mon obsession de tout vouloir fermer parce que tout me semblait moche autour. La laideur est présente partout dans les villes. Alors, plutôt que de lui tourner le dos et à défaut de pouvoir réparer les dégâts, nos interventions peuvent, peut-être, introduire quelque chose d'autre et soulager autant que possible les situations. Et plutôt que de faire un objet esseulé, insolite, il s'agit de faire le choix de rentrer dans le jeu des choses, même indigentes, présentes à côté. Objet de critique elle aussi, cette seconde maison est très bien acceptée aujourd'hui.

- Dominique Machabert. Voyons à présent la maison Van Middelem-Dupont à Oudenburg près d'Ostende en Belgique (1997-2003). On y reconnaît votre présence, votre sensibilité très subtile, presque brève, qui retourne pourtant l'intervention, la décolle de son milieu. 


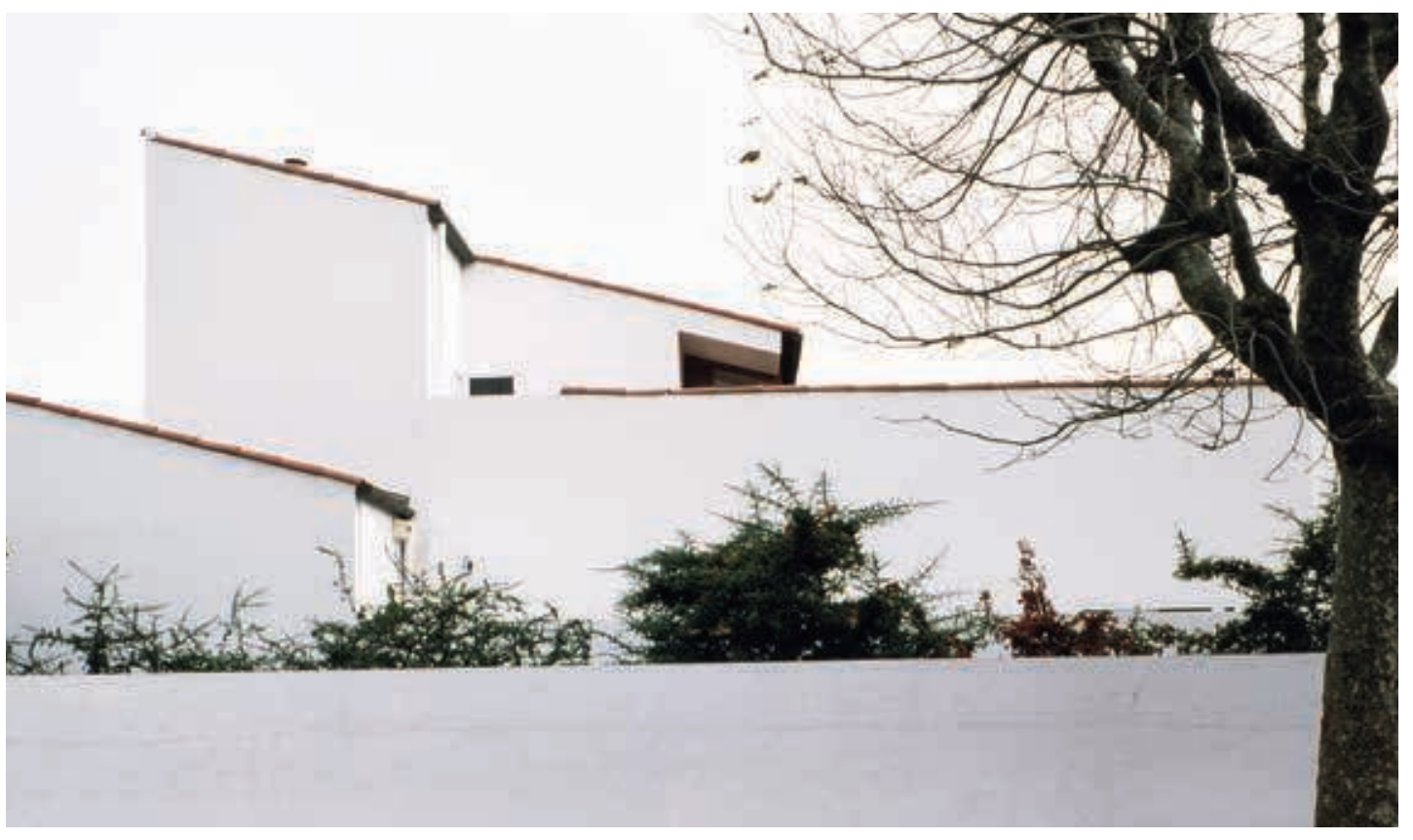

13. Álvaro Siza, maison Alves Santos, Póvoa de Varzim, Porto, 1964-1970, photographie de Luis Ferreira Alves.
C'est tout le contraire d'une réplique parfaite d'un état antérieur de la situation. Une intervention qui, plutôt que de s'y fondre, s'en dégage.

Pourriez-vous en dire plus à propos de ce projet afin que nous puissions en tirer un peu d'argument sur cette capacité à se faire "l'autre "; votre aptitude à restituer cette fois un certain caractère, celui de la Flandre, en Belgique?

- Álvaro Siza. Concernant cet ensemble de bâtiments en Belgique, rappelons qu'il s'agit pour partie d'un travail de restauration d'éléments existants. Il s'agissait d'un complexe agricole, d'une ferme dans la campagne flamande, avec des vaches... Il y avait une grange, une petite maison où habitait encore le chef de famille à l'époque, un patio. C'était comme cela [il dessine]. Il y avait une voie pour circuler, assez étroite, avec un cours d'eau à côté. Ce que j'ai fait ce sont deux bâtiments autour d'une cour ouverte. De strictement nouveau, il n'y a que ces deux bâtiments. Les autres ont été réhabilités (fig. 15).

Il y avait en effet, bien présent et qui préexistait, quelque chose du caractère dont vous parlez. Aussi, avec le changement de programme et de destination de cet ensemble devenu, sous l'effet des exigences de la vie moderne, un lieu pour vivre avec une petite galerie d'art intégrée dans un des bâtiments anciens, j'ai travaillé à prolonger, jusque dans le détail des bâtiments nouveaux, l'atmosphère générale qui rayonnait depuis le patio. Et il en va ainsi d'une espèce de domesticité perçue aussi de l'intérieur vers l'extérieur. Signalons toutefois qu'une petite activité agricole y perdure. Un bâtiment lui est toujours consacré. La présence d'animaux à l'extérieur rappelle la vocation d'origine du lieu, s'il le fallait.

- Dominique Machabert. À quoi cela tenait-il de transformer les lieux sans les bouleverser?

- Álvaro Siza. C'est mon métier, j'y suis obligé. En fait il s'agissait d'organiser les nouveaux volumes avec les anciens à partir du sol et de la façon de l'occuper. Il y avait un patio et j'ai 


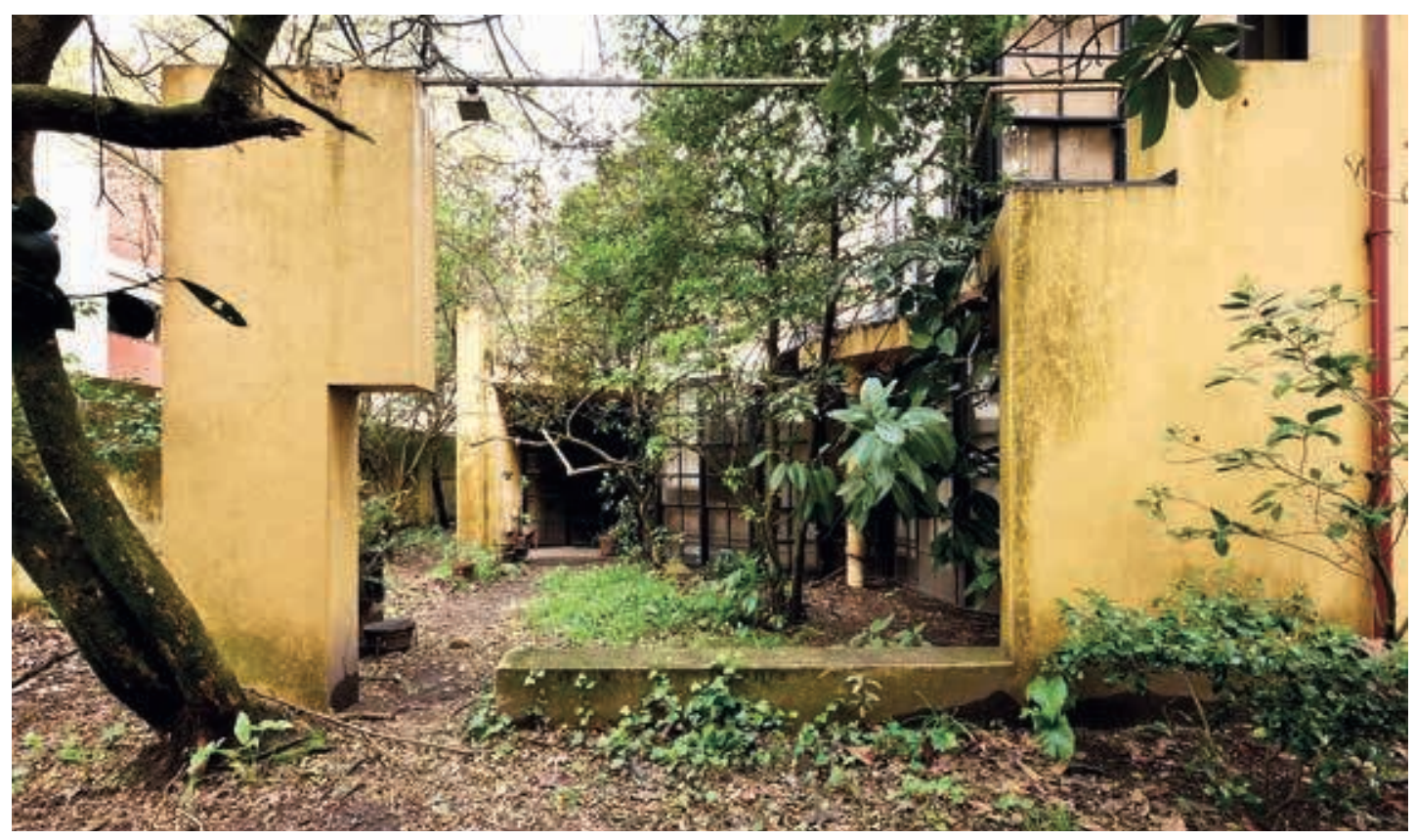

ajouté un pavillon avec un patio aussi, de façon à trouver les 14. Álvaro Siza, maison Beires, Póvoa de Varzim, Porto, 1973-1976, bonnes proportions. Ensuite on a fait une piscine. Il y a la possibilité de déjeuner dehors en rapport avec telle fenêtre, une photographie de Luis Ferreira Alves. fenêtre un peu «spéciale » qui élargit le champ de vision. On peut voir les vaches. La double présence des activités agricole et résidentielle, avec la galerie d'art, est très riche je trouve.

La question des matériaux s'est alors posée. Vous constatez que les toits sont en pente, à cause de la neige. Mis à part cela on remarque que, d'un côté, il y a du bois ; et, de l'autre, de la brique. À tel endroit, j'ai décidé d'employer un bois plus sombre, naturel. À tel autre, j'aurais préféré mettre du plomb mais la réglementation $n^{\prime} y$ autorisant pas, nous avons mis du zinc. Donc nous nous retrouvons tantôt avec des tuiles, tantôt avec du métal. Et même les détails, tous les détails, ont cet aspect que je dirais « moderne » bien que je trouve un peu simple de le dire comme cela. II ne s'agit donc pas d'une imitation des bâtiments anciens mais nous sommes dans l'esprit. C'est dans le rapport des volumes entre eux, comme dans une continuité de ce qui existait, que j'ai pu trouver ce caractère.

- Dominique Machabert. Je pense à la célèbre chaise de type Thonet qu'Adolf Loos a redessinée. Il semble qu'il ne s'agit que d'un bougé mais le pas est immense. Comme pour la maison Van Middelem-Dupont qui vous fait dire, quand même, à défaut de trouver un mot plus juste, qu'elle est « moderne »?

- Álvaro Siza. Pour ce projet comme pour d'autres bien différents, et dans les cas où il s'agit de sites habités, pas du désert, je dirais que j'ai cherché à ce qu'on puisse dire de l'intervention qu'elle a toujours été là - une forme d'intemporalité qu'Adolf Loos a recherchée et obtenue dans son architecture comme dans son mobilier. Dans sa formidable œuvre écrite - dans le contexte de l'Autriche du début de Xxe siècle - alors qu'il s'opposait au décor admirable de l'époque qu'il jugeait assez décadent, Adolf Loos explique qu'il dessinait des meubles anciens faits par des artisans traditionnels qui suivaient ses indications à la lettre, car il estimait 


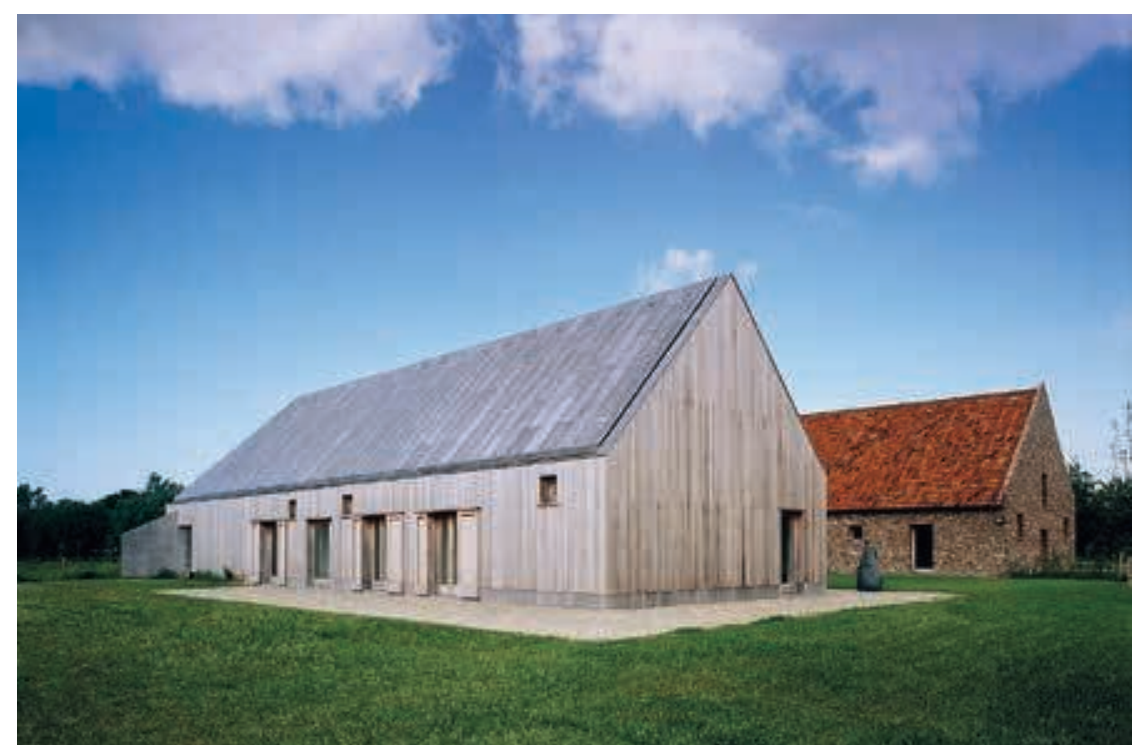

15. Álvaro Siza, maison Van Middelem-Dupont, Oudenburg (Belgique), 1997-2003, photographie de Roland Halbe. qu'il se devait d'accompagner les occupants du lieu, avec leurs usages, et une façon bien à eux de l'occuper. Porté par le souffle du renouveau, il gardait cependant à l'esprit l'idée d'une certaine continuité, recherchant toujours des références historiquement identifiables ou pouvant être comprises.

Alors, à n'en pas douter, c'est bien d'une chaise de type Thonet tout à fait identifiable, tout à fait banale qu'il s'agit (fig. 16). Mais lui, en la redessinant, il en fait une chaise pas banale du tout. On peut tirer de là une leçon sur la chose banale qui n'est pas aussi inerte qu'il n'y paraît, mais qui agit dans la continuité, dans l'histoire.

- Dominique Machabert. Je vois cette façon chez vous aussi de ne pas trop y toucher, de pousser à peine la chose banale, de la déplacer de façon à la dégager d'un statut de relative indifférence ou d'anonymat. C'est pourquoi j'ai parlé d'une sorte de retrait d'identité chez vous. Il n'empêche que, comme pour la maison Van Middelem-Dupont, la transformation est fondamentale. Un acte qui clôt et qui ouvre.

- Álvaro Siza. C'était le propos essentiel de Távora et son engagement notamment dans I'« Enquête ». Et c'est aussi le propos de son opuscule intitulé : « Le problème de la maison portugaise ».

- Dominique Machabert. Vous-même, avez-vous participé à I'« Enquête »?

- Álvaro Siza. Non.

- Dominique Machabert. Pourquoi ?

- Álvaro Siza. Parce que je n'ai pas été jugé assez apte. J'ai d'abord été un étudiant assez médiocre. Et je n'ai manifesté d'intérêt pour l'architecture qu'à compter du moment où Távora est devenu mon professeur. Távora se penchait sur nos travaux mais pas pour 
en dénoncer les faiblesses. II repérait et manifestait plutôt la part de recherche qui pouvait se trouver dans chacun de nos résultats, fussent-ils moyens ou médiocres, comme dans mon cas. Une fois, j'ai même obtenu la deuxième médaille qui était la plus haute distinction. J'ai su qu'il y avait eu une grande discussion entre professeurs concernant cette attribution. Távora a défendu mon projet y voyant, plutôt que des faiblesses évidentes, l'esprit de recherche qui l'avait animé.

Il y eut bien d'autres moments qui témoignent de mes débuts laborieux. J'étais en deuxième année et je me souviens de Carlos Ramos qui était alors directeur de l'école et un professeur d'un très haut niveau. Je le revoie roulant sa cigarette, puis tirant de sa poche un petit ciseau avec lequel il coupait les deux extrémités, tac, tac. Et c'est seulement après qu'il se mettait à parler. Mais devant mon travail, comprenant aussitôt mon absence de bagage et de notion, il eut la délicatesse de ne pas le juger et m'invita alors à me rendre dans une librairie et à faire l'achat de quelques revues d'architecture. Ce que je fis, accompagné par mon père. Vous voyez d'ici le tableau : un grand garçon inscrit en cours supérieur parti acheter des revues en compagnie de son père! Parmi les quatre revues que j'achetai, il y en avait une sur Aalto que je ne connaissais pas et que presque personne ne connaissait d'ailleurs. J'ai encore à l'esprit la grimace que firent mes collègues, tous bien plus au courant que moi, et dont certains étaient même des fils d'architecte, en me voyant arriver avec mes revues. Aalto m'avait fait une telle impression que la réaction réprobatrice de mes collègues m’avait plongé dans un grand désarroi. Décidemment je ne comprends rien, me disai-je.

- Dominique Machabert. Il y avait les bons élèves...

- Álvaro Siza. Et les ignorants comme moi, seul à aimer Aalto. Disons que j'étais émerveillé par ce que j'avais découvert, pour des raisons qui n'étaient alors qu'esthétiques, comme pour la peinture. Mes collègues avaient des fondements critiques plus assurés à cette époque où Le Corbusier occupait à peu près tout le champ.

- Dominique Machabert. Peut-on dire que vous avez gardé cette grille de lecture?

- Álvaro Siza. Oui, et même si elle n'est plus la seule, je dois dire que mon rapport aux arts demeure une référence sur laquelle je m'appuie toujours. Cela explique, concernant Aalto par exemple, que je n'en ai d'abord compris qu'une partie. Tout son travail sur I'habitat social des années 1930, pas tant pour les volumes ou pour les formes, que pour les espaces, m'était passé complètement au-dessus à l'époque. Je ne voyais les choses que d'un point de vue esthétique. C'était très

16. Adolf Loos, chaise créée pour le café Museum (Vienne), vers 1889, hêtre courbé, teinté et verni, cannage, manufacture Jacob et Josef Kohn (Vienne), Paris, musée d'Orsay, inv. nº OAO 573.




incomplet. Mais la lecture, plus tard, des revues et des textes critiques de Sigfried Giedion, notamment, m’ont beaucoup éclairé et ont élargi mon champ.

- Dominique Machabert. À l'occasion d'un autre entretien vous disiez qu'au cours de vos premières années de formation, vous vous êtes d'abord tenu distant de la pensée et de l'architecture de Le Corbusier archidominante alors.

- Álvaro Siza. J'étais surtout très ignorant jusqu'à ce que des circonstances heureuses m'éclairent et fassent sauter tous les lieux communs et les raccourcis à propos de Le Corbusier et de son œuvre, qui est essentielle.

- Dominique Machabert. Je m'explique votre travail et votre architecture aussi pour l'intérêt que vous portez aux choses moins savantes. Je pense à votre goût pour William Saroyan, écrivain américain que vous préférez à William Faulkner par exemple. Je pense à Blaise Cendrars lorsque vous évoquez son rapport direct aux choses les plus simples, les plus vivantes semblant même ne pas s'encombrer beaucoup avec les conventions littéraires.

- Álvaro Siza. Il y a chez ces deux-là une façon d'attraper l'atmosphère...

Propos recueillis par Dominique Machabert,

Porto, août 2019. 


\section{NOTES}

1. Fernando Távora (1923-2005) : architecte, il fonde, avec Viana do Lima, le groupe portugais des CIAM (Congrès international de l'architecture moderne). Auteur d'une œuvre remarquable qui allie tradition et modernité, il est la figure emblématique de l'école de Porto.

2. Arthur Rimbaud, "Les Poètes de sept ans ", 26 mai 1871, dans une lettre à Paul Demeny datée du 10 juin 1871.

3. Antonio Tabucchi, Pereira prétend : un témoignage, Bernard Comment (trad. fr.), Paris, Christian Bourgois, 1995 [éd. orig. : Sostiene Pereira: una testimonianza, Milan, Feltrinelli, 1994].

4. L'« Enquête sur l'Architecture populaire au Portugal » réalisée par des équipes d'architectes entre 1955 et 1960 (publiée en 1961 et réédité en 1988, 1988 et 2004, sous le titre Arquitectura popular em Portugal, Lisbonne, Sindicato nacional dos arquitectos, 1961, 2 vol.) est née de l'initiative du Syndicat national des architectes et de son président Francisco Keil do Amaral. Elle présente de façon rigoureuse la situation réelle du pays, étudiée finement jusque dans ses confins. Contrariant le dessein du régime de Salazar désireux de porter ses efforts sur une architecture « folklorique » et nationaliste, I'« Enquête » montrait une voie possible et argumentée située entre la modernité et la véritable tradition portugaise, vers laquelle l'école de Porto s'orienterait ensuite.

5. Fernando Távora, "O problema da casa portuguesa ", Aléo, année IV, série IV, n 9, 10 novembre 1945, reparu dans Luís Trigueiros et Fernando Távora, Fernando Távora, Lisbonne, Blau, 1993, p. 11-13.

6. Eszter Steierhoffer (dir.), "Coin, îlot, quartier, villes : Álvaro Siza à Berlin et à La Haye ", exposition organisée à Montréal, au Centre canadien d'architecture, en 2015 [URL : cca.qc.ca/fr/evenements/3509/coin -ilot-quartier-villes-alvaro-siza-a-berlin-et-a-la-haye].

7. Le label IBA ("Internationale Bauausstellung» ; "Exposition internationale d'architecture ») date de 1901. L'objectif était alors d'exposer les nouveautés architecturales, notamment en matière de logements. Depuis I'IBA de Berlin, en 1987, consacrée à la réhabilitation et plus largement à la rénovation urbaine, les expositions sont désormais conçues comme des instruments d'aménagement qui, à travers des projets ciblés, engagent des mutations urbaines et territoriales.

8. Álvaro Siza a reçu le Grand Prix d'architecture de I'Académie des beaux-arts - prix Charles Abella 2019, le 27 novembre 2019 ; il avait été invité à prononcer un discours sous la coupole du palais de l'Institut de France le 9 octobre 2019.

9. Philip L. Goodwin, Brazil Builds. Architecture New and Old / Construção brasileira: arquitetura moderna e antiga (1652-1942), G. E. Kidder Smith (photographies), cat. exp. (New York, The Museum of Modern Art, 1943), New York, The Museum of Modern Art, 1943.

10. Álvaro Siza, Viagem sem Programa: Interview and Portraits, Bologne, Red Publishing, 2012. On peut aussi citer l'exposition du même nom qui eut lieu en 2018, à l'atelier Aires Mateus (Lisbonne).

11. Alvar Aalto, "La truite et le ruisseau » (Domus, 1947), dans La table blanche et autres textes, Anne
Colin du Terail (trad. fr.), Marseille, Éditions Parenthèses, 2012, p. 109 et suiv.

12. Jorge Luis Borges, L'auteur et autres textes (1960), Roger Caillois (trad. fr.), Paris, Gallimard, 1965.

13. Álvaro Siza, Imaginer l'évidence, Vittorio Gregotti et Marc Barani (préfaces), Dominique Machabert (trad. fr.), Marseille, Parenthèses, 2012. 